\title{
Memory for spatial location: Cue effects as a function of field rotation
}

\author{
Sylvia Fitting, Douglas H. Wedell, and Gary L. Allen \\ University of South Carolina, Columbia, South Carolina
}

\begin{abstract}
We developed theoretical extensions of Huttenlocher, Hedges, and Duncan's (1991) category-adjustment model of human spatial memory to incorporate the use of fuzzy boundaries and cue-determined prototypes. In two experiments, people reproduced locations of dots in a circle, while the number of external reference cues varied. In Experiment 1, the task field was stable and results were consistent with the use of fixed categories unaffected by number of cues. In Experiment 2, the task field was made dynamic by rotation on most trials, with results evaluated for nonrotation trials. The large cue effects observed for angular bias were consistent with the proposed cue-based fuzzy-boundary model. Large cue effects were also observed for absolute error, consistent with a model in which proximity to cues predicts stability of memory. Results point to the key role of orientation to the task environment in determining whether categorical encoding is based on cues.
\end{abstract}

A striking feature of spatial memory for location is that it is highly dependent on the frame of reference adopted at encoding. The spatial cognition literature describes at least three frames of reference used to code memory for location: geometric, cue based, and egocentric (Allen, Kirasic, Rashotte, \& Haun, 2004; Cheng, 1986; Huttenlocher, Hedges, \& Duncan, 1991; Mou \& McNamara, 2002; Wedell, Fitting, \& Allen, 2007). Both geometric and cue-based frames are allocentric in nature, since locations are coded using configural relationships among external features (O'Keefe \& Nadel, 1978; Tolman, 1948). The egocentric frame of reference, on the other hand, codes object locations in terms of directions (right, left, up, down, forward, and backward) and distance from the viewer, with a definable axis of orientation along with distance information (Gallistel, 1990).

Different experimental paradigms provide the basis for distinguishing among the use of these different frames of reference. The experiments we report here were designed to distinguish between the use of cue-based and cueindependent frames of reference. Cue-based representations may be considered examples of an allocentric coordinate system relating location to environmental cues. An empirical example of this type of representation derives from studies of the Morris water maze, in which place memory is determined by spatial relations among distal cues and to-be-remembered locations (Morris \& Parslow, 2004; Nadel, 1990). The Morris water maze task forces the cue-based referencing of location, because the entry point is systematically varied so that the participant cannot simply remember a direction and distance from the starting point. Cue-independent representations may be derived from either egocentric or geometric frames, which do not code location relative to environmental cues.
The experiments we report in this article investigated the role of cues in a simple 2-D spatial location task. In our task, a location could be remembered using a cue-based or a cue-independent encoding system, with the pattern and nature of errors expected to differ as a result of the encoding framework. We analyze the results by developing quantitative models that describe the errors and bias that result from these two encoding schemes. For these purposes, we adopted the task of locating a dot within a circular task field, for which a clear model of bias in memory has been developed by Huttenlocher and colleagues (Huttenlocher, Hedges, Corrigan, \& Crawford, 2004; Huttenlocher et al., 1991). These researchers have provided good evidence that errors in memory in this task reflect the use of category prototypes to resolve uncertainty, with each prototype representing a central location within one of the four quadrants of the circular task field. What is unclear, on the basis of previous research, is the degree to which these prototypes that are hypothesized to rely on intrinsic vertical and horizontal axes can be shifted by cues. For example, the geometric properties of the circular task field, along with surrounding background information, may fix the location of the dot in a cue-independent frame of reference, with prototypes determined by a natural division of the circular region. On the other hand, added external reference cues may be capable of shifting the orientation from a cue-independent to a cue-based frame of reference.

To distinguish between these possibilities, we introduced two new features to the task. The first is the inclusion of external cues. If a cue-based frame of reference is used, available cues should be incorporated into the representation and influence prototype locations, biasing memory for location. On the other hand, if a cue-independent frame of reference

S. Fitting, fitting@sc.edu 
is used, the cues would be irrelevant, and performance on the task should remain unchanged across cue conditions. The second new feature we introduced concerns whether the task field maintains a stable orientation or whether it is dynamically rotated across trials. Here, the external cues served to indicate the degree of rotation. In the same way as varying starting points in the water maze task (Morris, Garrud, Rawlins, \& O'Keefe, 1982), the introduction of rotation was meant to induce a cue-based frame of reference. Because the cue rotation indicated how much the participant must rotate the location of the object, orienting the object relative to the cues seemed likely. A key comparison between conditions would be what happened on nonrotation trials, these being identical in both conditions.

We hypothesized that the use of a cue-based reference system would depend on the uncertainty of orientation to the task field. With a stationary task field orientation, uncertainty about orientation is clearly low; hence, a frame of reference with fixed geometric categories is likely to be imposed, regardless of the availability of peripheral cues. Furthermore, the ease of maintaining spatial information with a stationary task field may render the presence of cues irrelevant and uninformative; they may, therefore, be largely ignored. In contrast, with a more dynamic task field in which rotation of orientation occurs on a regular basis, uncertainty about orientation is high. Under conditions of high uncertainty, available peripheral cues should gain prominence in establishing a frame of reference for the task. It is well established that observers can track spatial location when response fields are rotated relative to the observer's initial view (Shepard \& Metzler, 1971). However, it is not known how rotation of the circular field affects categorical coding, nor is it clear whether the possibility of task field rotation per se affects such coding (as when memory trials requiring rotation are intermixed with trials requiring no rotation).

To address these issues, we designed two experiments that involved modeling responses from observers who completed a series of trials requiring memory for a briefly presented location within a circular field. In Experiment 1, the task and response field were static and the number of external peripheral cues was varied (zero, one, or three). In Experiment 2, participants were required to accommodate rotation of the response field after initial encoding and the number of external peripheral cues was again varied (one and three). A key to understanding the implications of adopting the different frames of reference revolves around how predicted error or bias in memory is influenced by the frame of reference. We therefore describe three models that are applicable and how these were developed on the basis of prior work. Modeling the data will be a critical determinant of understanding how cues are incorporated into the spatial representation of the object location.

\section{Model Development}

Our model builds on the basic category-adjustment model described by Huttenlocher et al. (1991), which provides a good approximation to the bias typically observed in the dot location task. The category-adjustment model is based on a four-quadrant encoding scheme and therefore uses a cue-independent frame of reference. Our model development first introduces the use of fuzzy boundaries to explain the diminished bias effects found for objects located near category borders. This model is similar to the uncertainboundary version of the model developed by Huttenlocher et al. (1991), except that it uses a more flexible prototype recruitment function that allows us to develop a cue-based version of the model that does not require prior specification of boundary locations. Our cue-based fuzzy-boundary model then incorporates the idea that category prototypes may be based on cue locations. We demonstrate that the pattern of bias is markedly different for the cue-based versus fixed-quadrant approaches. Our modeling is restricted to angular bias, since this is the key variable we expect to be moderated by the inclusion of cues.

\section{The Basic Category-Adjustment Model}

Huttenlocher et al. (1991) described the interplay between two types of information in their category-adjustment model: a fine-grain value and a categorical value. Note that the navigation literature incorporates a similar distinction between high-fidelity and coarser information used in egocentric or allocentric orientations (Burgess, Spiers, \& Paleologou, 2004; Easton \& Sholl, 1995; Mou, McNamara, Valiquette, \& Rump, 2004; Wang \& Spelke, 2000). The two-level approach to spatial memory appears to be well established across a number of different spatial memory paradigms. The memory of the fine-grain information is centered on the actual location, whereas the memory of the categorical information is centered on the category prototype location. Biases in responding arise from combining information from the two levels to produce an estimate, conceived as a weighted average of the relatively shortlived fine-grain values and more robust central category prototypes (with some versions of the model including the influence of truncation at category boundaries). The functional outcome of weighting categorical information in estimation is a predictable bias toward the category prototypes, posited to correspond to the centers of the geometrically determined categories (Huttenlocher et al., 1991). Although weighting of categorical information can produce a pattern of bias in estimation, it also reduces overall error in estimation, just as centering estimates on the mean minimizes squared error in standard regression models. Thus, Huttenlocher et al. (1991) have emphasized the rational and adaptive nature of this memory process.

Because bias in estimation was central to our investigation, we will present a series of formal models that were used to generate testable predictions. According to the category-adjustment model of Huttenlocher et al. (1991), the expected value of the response in an estimation task, $E[R]$, can be characterized as a weighted average of finegrain and categorical information described by the following equation:

$$
E[R]=\lambda \mu+(1-\lambda) p,
$$

where $\mu$ is the mean of the distribution of fine-grain memory values for the object, assumed to be unbiased and, hence, equated with the true location of the object. 
Similarly, $p$ is the mean of the distribution of prototype locations for the relevant category. The parameter $\lambda$, which varies from 0 to 1 , represents the relative weight of the fine-grain information. Bias is determined by subtracting the actual value from the response; thus, the expected bias is characterized by the following equation:

$$
E[\text { Bias }]=E[R]-\mu=\lambda \mu+(1-\lambda) p-\mu .
$$

In general, the greater the uncertainty concerning finegrain information, the less the weight of the fine-grain information (i.e., $\lambda$ decreases). In Equations 1 and $2, \lambda$ is a constant and therefore reflects the assumption that uncertainty does not vary across locations within a category. Following Huttenlocher et al. (1991), we represent the two components of the responses being modeled within a polar coordinate system, a point being represented by its radial distance and the angular direction from the center of the circle. Although the model applies to both radial and angular components, the primary focus of our experiments was on angular bias.

Although the applicability of the category-adjustment model has been tested across different tasks, it was originally formulated for the task of locating a single dot within a circle, as adapted from Nelson and Chaiklin (1980). Figure 1A illustrates the model's prediction for this task. Following Huttenlocher et al. (1991), we assume that the participant imposes a categorical structure that divides the circle into four quadrants along the vertical and horizontal axes. As is shown in Figure 1, estimates are unbiased when stimuli are located at category prototypes, here assumed to be the center of the four quadrants of the circle (i.e., Quadrant 1 extends from $0^{\circ}$ to $90^{\circ}$ with midpoint at $45^{\circ}$, Quadrant 2 from $90^{\circ}$ to $180^{\circ}$ with midpoint at $135^{\circ}$, Quadrant 3 from $180^{\circ}$ to $270^{\circ}$ with midpoint at $225^{\circ}$, and Quadrant 4 from $270^{\circ}$ to $360^{\circ}$ with midpoint at $315^{\circ}$ ). Conversely, bias is maximized near the boundaries of each of the four quadrants, where the deviation from the prototype is maximal (i.e., near $0^{\circ}, 90^{\circ}, 180^{\circ}$, and $270^{\circ}$ ).

\section{The Fixed-Quadrants Fuzzy-Boundary Model}

One problem with the basic category-adjustment model is that a nonlinear bias function is sometimes observed. For example, Haun, Allen, and Wedell (2005) found nonlinear bias in incline and azimuth estimation tasks within a small-scale environment, with bias decreasing near the extreme angles of $0^{\circ}$ and $90^{\circ}$, which might be considered the boundaries of the category in their experiment. They argued that one cause of the reduced bias was the tendency to recruit prototypes from adjacent categories for targets near the border, resulting in biases' canceling out. Because ambiguity concerning the relevant prototype is greatest at category boundaries, they described their model as a fuzzy-boundary model. This model is consistent with the uncertain-boundary version of the categoryadjustment model proposed by Huttenlocher et al. (1991) but differs in ways we describe at the end of this section.

The fuzzy-boundary models we describe here differ from the Haun et al. (2005) model in that they are not limited to two categories and are adapted to the polar coordinate representation. Our fixed-quadrants fuzzy-boundary model uses a prototype recruitment function that is based on the relative similarity of the stimulus angle to the midpoints of the four quadrant-specified categories of the cue-independent representation. For our similarity function, we employ the often used exponential decay function (Shepard, 1987), in which similarity falls off very rapidly with increased distance. When applied to the cueindependent frame of reference (i.e., boundaries at $0^{\circ}, 90^{\circ}$, $180^{\circ}$, and $270^{\circ}$ ), the probability of prototype recruitment can be described as follows:

$$
\operatorname{Pr}\left(p_{j} \mid \mu\right)=\frac{\exp \left[-c\left|\mu-.5\left(t_{\min , j}+t_{\max , j}\right)\right|\right]}{\sum \exp \left[-c\left|\mu-.5\left(t_{\min , k}+t_{\max , k}\right)\right|\right]},
$$

where similarity is calculated relative to the midpoint of the category, using the average of the lower boundary $\left(t_{\min }\right)$, and upper boundary $\left(t_{\max }\right)$, and $c$ is a sensitivity parameter that represents the sharpness of the boundary (the lower the value of $c$, the fuzzier the boundary). By using the midpoints between a priori boundaries, Equation 3 represents a fixed-quadrants fuzzy-boundary model. Note that because of the polar coordinate system, angles that are in the first and fourth quadrants are incorrectly seen as distant to one another (i.e., $1^{\circ}$ and $359^{\circ}$ are seen as $358^{\circ}$ apart rather than $2^{\circ}$ apart). Thus, to properly apply this model, we include two additional midpoints corresponding to the lowest valued midpoint plus $360^{\circ}$ (i.e., $45^{\circ}+360^{\circ}=405^{\circ}$ ), along
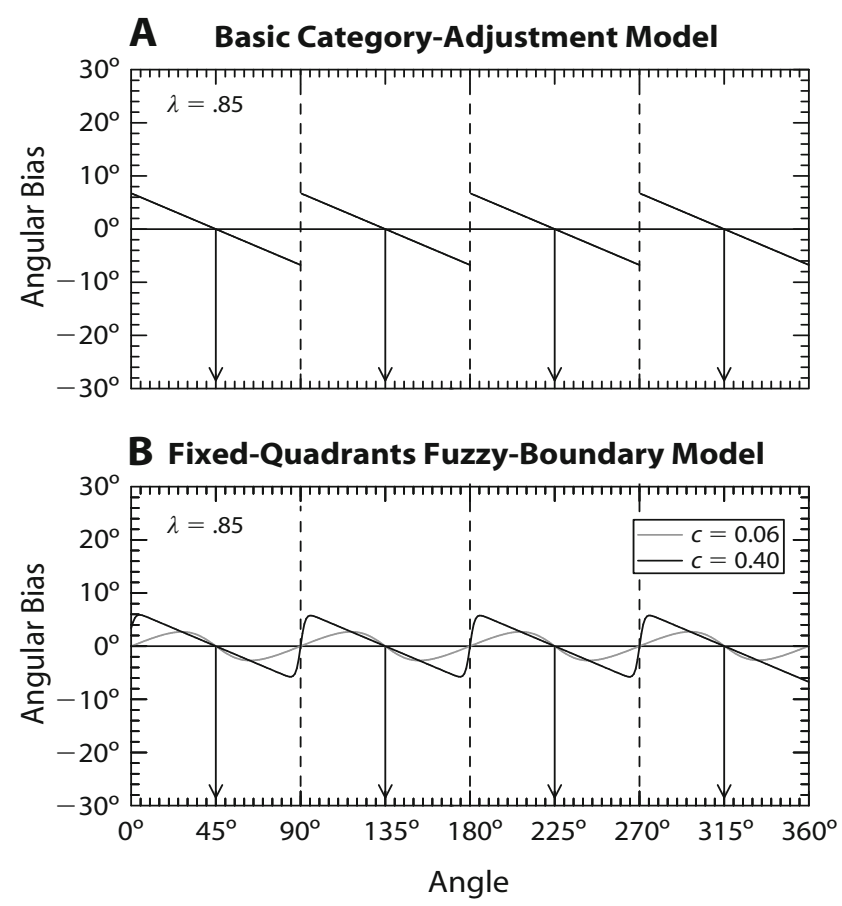

Figure 1. (A) Category-adjustment model of Equation 2 applied with boundaries fixed along the axes at $0^{\circ}, 90^{\circ}, 180^{\circ}$, and $270^{\circ}$, and prototypes at the midpoint of each quadrant at $45^{\circ}$ $135^{\circ}, \mathbf{2 2 5}^{\circ}$, and $315^{\circ}$. (B) Fixed-quadrants fuzzy-boundary model of Equations 2 and 3 with the same fixed boundaries and quadrant prototypes. Weighting of fine-grain information is indicated by $\lambda$, and the sensitivity parameter by $c$. 
with the highest valued midpoint minus $360^{\circ}$ (i.e., $315^{\circ}$ $-360^{\circ}=-45^{\circ}$ ). These virtual midpoints are necessary to allow the model to recruit the Quadrant 1 prototype for Quadrant 4 angles and vice versa. In other words, the recruitment of prototypes from either clockwise or counterclockwise rotation requires the addition of these "virtual" category midpoints. (Note also that since a single radial prototype is typically assumed for all categories, the fuzzyboundary model is applied only to angular estimation.) ${ }^{1}$

Figure 1B shows the predicted pattern of bias from this fixed-quadrants fuzzy-boundary model that combines Equations 2 and 3 for the dot spatial location task. Like the basic category-adjustment model shown in Figure 1A, fixed boundary locations are assumed at $0^{\circ}, 90^{\circ}, 180^{\circ}$, and $270^{\circ}$. Although bias at first increases as the angle moves away from the prototype location, it later decreases as it approaches the boundary because, near the boundary, the angle is increasingly likely to recruit the prototype from the adjacent category; hence, bias is added in the opposite direction. Although the prototype locations are shown at the midpoints of the categories, the model combining Equations 2 and 3 does not require this, but, rather, requires only that the prototype lie somewhere within the category boundaries. Finally, note that as the value of the sensitivity parameter increases, the fuzzy boundaries sharpen, so that the predicted pattern becomes more like that of the basic category-adjustment model.

There are two main differences between the fuzzyboundary version of the model developed here and the uncertain-boundary version of the model developed by Huttenlocher et al. (1991). First, the uncertain-boundary version is based on prior determination of boundaries. The fuzzy-boundary model infers boundary locations from similarity to category centroids and, therefore, does not need to specify boundaries a priori. Although the fixedboundaries version of the model does use a priori defined boundaries, the option of inferring boundaries within this framework provides the model the needed flexibility to model data with unknown boundaries, as is discussed below. Second, the uncertain-boundaries version uses a normal distribution of error at the boundary to model prototype recruitment, whereas the fuzzy-boundary model utilizes a negative exponential similarity function based on prototype or midpoint locations to guide prototype recruitment. In general, because the differences between the models in the fixed-quadrants version are minimal, they lead to very similar predictions. The real value of the fuzzyboundary model is found in its flexibility, which allows it to be applied to cases in which the number of prototypes and their locations are not known a priori. This point is developed in the following section.

\section{The Cue-Based Fuzzy-Boundary Model}

The focus of the studies reported in this article is on the potential influence of cues located at different points along the perimeter of the circle on bias in estimation. The most straightforward extension of the fuzzy-boundary version of the category-adjustment model involves the assumption that the cues serve as prototypes; this means that we can no longer use the fixed boundaries described by a cue-independent frame of reference $\left(0^{\circ}, 90^{\circ}, 180^{\circ}\right.$, and $270^{\circ}$ ). Instead, we infer boundaries by assuming that they fall at equal distances from the category prototypes. Accordingly, we alter the prototype recruitment equation to reflect the relative similarities of the stimulus to the various prototypes rather than to the midpoints of the categories, as follows:

$$
\operatorname{Pr}\left(p_{j} \mid \mu\right)=\frac{\exp \left(-c\left|\mu-p_{j}\right|\right)}{\sum \exp \left(-c\left|\mu-p_{k}\right|\right)} .
$$

Once again we include "virtual" prototypes for the lowest and highest categories so that recruitment may be conducted in a clockwise or a counterclockwise fashion. Note that even when there is only one prototype, there will be a "virtual" boundary created by the inclusion of these virtual prototypes. For example, consider the case in which the cue is located at $305^{\circ}$ so that the category prototype is also located at $305^{\circ}$. Virtual prototypes will be created at $665^{\circ}$ and $-55^{\circ}$. Given that similarity falls off exponentially, the virtual prototype at $665^{\circ}$ will have no impact, but the prototype at $-55^{\circ}$ will. Indeed, angles in Quadrant $1\left(0^{\circ}-90^{\circ}\right)$ will recruit this virtual prototype and therefore exhibit a negative bias. The point halfway between the prototypes at $305^{\circ}$ and $-55^{\circ}$ (i.e., at $125^{\circ}$ ) will then represent the virtual boundary in this single-prototype case.

Figure $2 \mathrm{~A}$ describes the predictions of this cue-based fuzzy-boundary version of the category-adjustment model for the one-prototype case (with the prototype at $305^{\circ}$ ). As is shown, the bias function crosses $0^{\circ}$ at two points, once at the prototype value of $305^{\circ}$ and once at the inferred boundary value of $125^{\circ}$. This striking pattern of bias is quite different from the usual pattern shown in Figure 1 and thus should be easily detected.

Figure 2B describes the predictions of the cue-based fuzzy-boundary model for the case in which there are three cues and therefore three prototypes, located at $p=$ $80^{\circ}, 170^{\circ}$, and $305^{\circ}$. Once again, two virtual prototypes are added: one at $p=440^{\circ}$ and the other at $p=-55^{\circ}$. These produce inferred boundaries at $237.5^{\circ}, 12.5^{\circ}$, and $125^{\circ}$. The pattern of bias generated by three prototypes and shown in Figure $2 \mathrm{~B}$ is thereby highly distinguishable from the pattern produced by one prototype and shown in Figure 2A. It is also quite distinct from those produced by the basic category-adjustment model and the fixed-quadrants fuzzyboundary model shown in Figure 1. It is useful to note that the pattern of bias shown in Figure 1B can be produced by the cue-based fuzzy-boundary model of Equations 2 and 4 if there are four prototypes located at $45^{\circ}, 135^{\circ}, 225^{\circ}$, and $315^{\circ}$. Finally, note that overall deviation from the unbiased values is much greater in the one-cue than in the three-cue condition. Therefore, the model predicts that absolute error will decrease as the number of cues increases.

\section{Overview of Experiments}

Previous research has demonstrated that when observers remember locations within a circular field, their memory is biased by implicit categories imposed on the circle (Huttenlocher et al., 1991) and consistent with either intrinsic or viewer-based frames of reference that uti- 


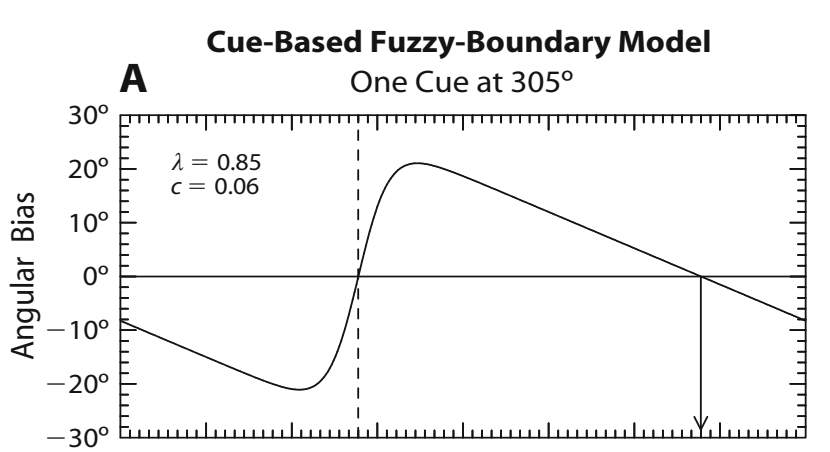

B Three Cues at $80^{\circ}, 170^{\circ}$, and $305^{\circ}$

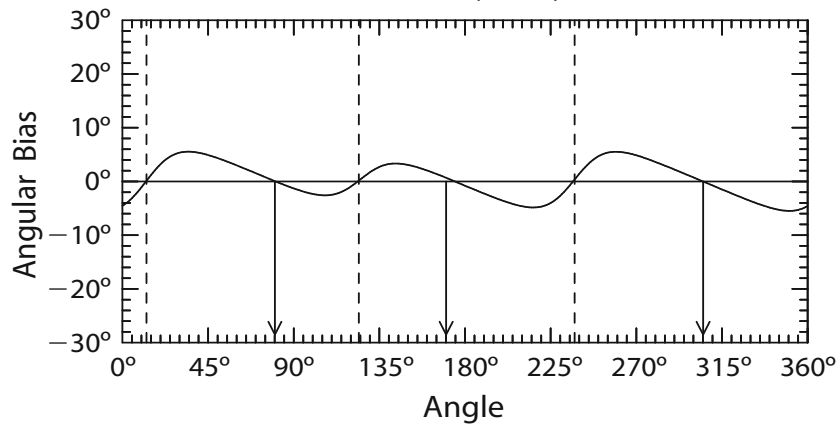

Figure 2. Predictions of the cue-based fuzzy-boundary model of Equations 2 and 4, with prototypes equated with cue location, probabilistic recruitment of prototypes across categories, and inferred boundaries located halfway between adjacent prototypes. (A) One cue case with the prototype at $305^{\circ}$ and the boundary at $125^{\circ}$. (B) Three prototypes located at $80^{\circ}, 1^{\circ}$, and $305^{\circ}$, with inferred boundaries at $237.5^{\circ}, \mathbf{1 2 . 5}^{\circ}$, and $125^{\circ}$. Weighting of fine-grain information is indicated by $\lambda$, and the sensitivity parameter by $c$.

lize vertical and horizontal boundaries. The key questions asked in our research concerned when and how external cues might influence estimates. Our model development demonstrated distinctly different patterns of bias for cuebased processing (Figure 2) and fixed-quadrants-based processing (Figure 1).

In Experiment 1, we examined whether the mere presence of cues is enough to warrant their use. In Experiment 2, we introduced rotation of the task field on the majority of trials to see whether participants would continue to use cue-independent categories on the nonrotation trials. In each experiment, we modeled the data using the three basic models described, in order to determine the sphere of applicability of these models across environments that differ in the presence of cues and in uncertainty of orientation.

\section{EXPERIMENT 1}

In Experiment 1, we examined the applicability of the different models described above to a spatial memory task similar to the one used by Huttenlocher et al. (1991). In contrast to the standard task, we varied the number of available cues along the circular region. The purpose of Experiment 1 was (1) to determine whether the tendency to use fixed geometrical categories based on a cue-independent frame of reference in a static, unchanged task field setting would still be present when reference cues were available and (2) to investigate the fundamental tenets of different versions of the category-adjustment model in this task.

On the basis of the report of an intrinsic frame of reference (Huttenlocher et al., 1991), we hypothesized that, in a static task field setting, participants would ignore available reference cues and would infer horizontal and vertical boundaries, imposing implicit quadrants on the circular field. This might result from using an egocentric frame of reference, in which the left-right and up-down division of the circle would arise out of the viewer's orientation. Alternatively, a lack of external cue use could also be explained by the operation of an autonomous geometric module in spatial coding processes. The dominance of geometric spatial coding appears to be present early in development (Hermer \& Spelke, 1996; Wang, Hermer, \& Spelke, 1999) and has further been supported by animal studies (Cheng, 1986).

Second, we hypothesized that estimates of spatial dot locations would include substantial categorical bias predicted by the different versions of the category-adjustment model, thus biasing responses toward the middle of those inferred quadrants. We hypothesized that a static task field setting would maintain the relevance of the fixed horizontal and vertical boundaries typically used in the location task. Coding of spatial locations would be determined by a cue-independent frame of reference with fixed geometrical categories bounded at $0^{\circ}, 90^{\circ}, 180^{\circ}$, and $270^{\circ}$.

The results of the estimation process were quantified, using three different measures. Accuracy was determined by absolute error as a distance measure between the actual and the remembered dot location. Several researchers (Montello, Richardson, Hegarty, \& Provenza, 1999; Schutz \& Roy, 1973) have pointed out that absolute error has the problem of conflating constant error, reflected in means, with random error, reflected in variance. However, we include it as an intuitive measure that incorporates error due to bias measures and error at the fine-grain level. Cue independence predicts no effects of cues on accuracy, whereas the cue-based fuzzy-boundary model implies greater accuracy with more cues (i.e., a reduction in absolute error). Bias tendencies were assessed by measures of angular bias and radial bias. Cue independence implies that neither of these measures should be influenced by number of cues. The cue-based fuzzy-boundary model implies differences in the patterns of angular bias for the different conditions, as shown in Figure 2. These data will also provide a test between the basic categoryadjustment model and the fuzzy-boundary versions, in that only the fuzzy-boundary versions predict reduction of angular bias near category boundaries.

\section{Method}

\section{Participants}

Sixty-two undergraduate students from the University of South Carolina psychology department participant pool took part in the experiment in exchange for course credits.

\section{Task}

All participants attempted to reproduce the locations of 32 dots in a circular region presented on a computer display, as illustrated in Figure 3. The 32 targets were distributed over the circular area 


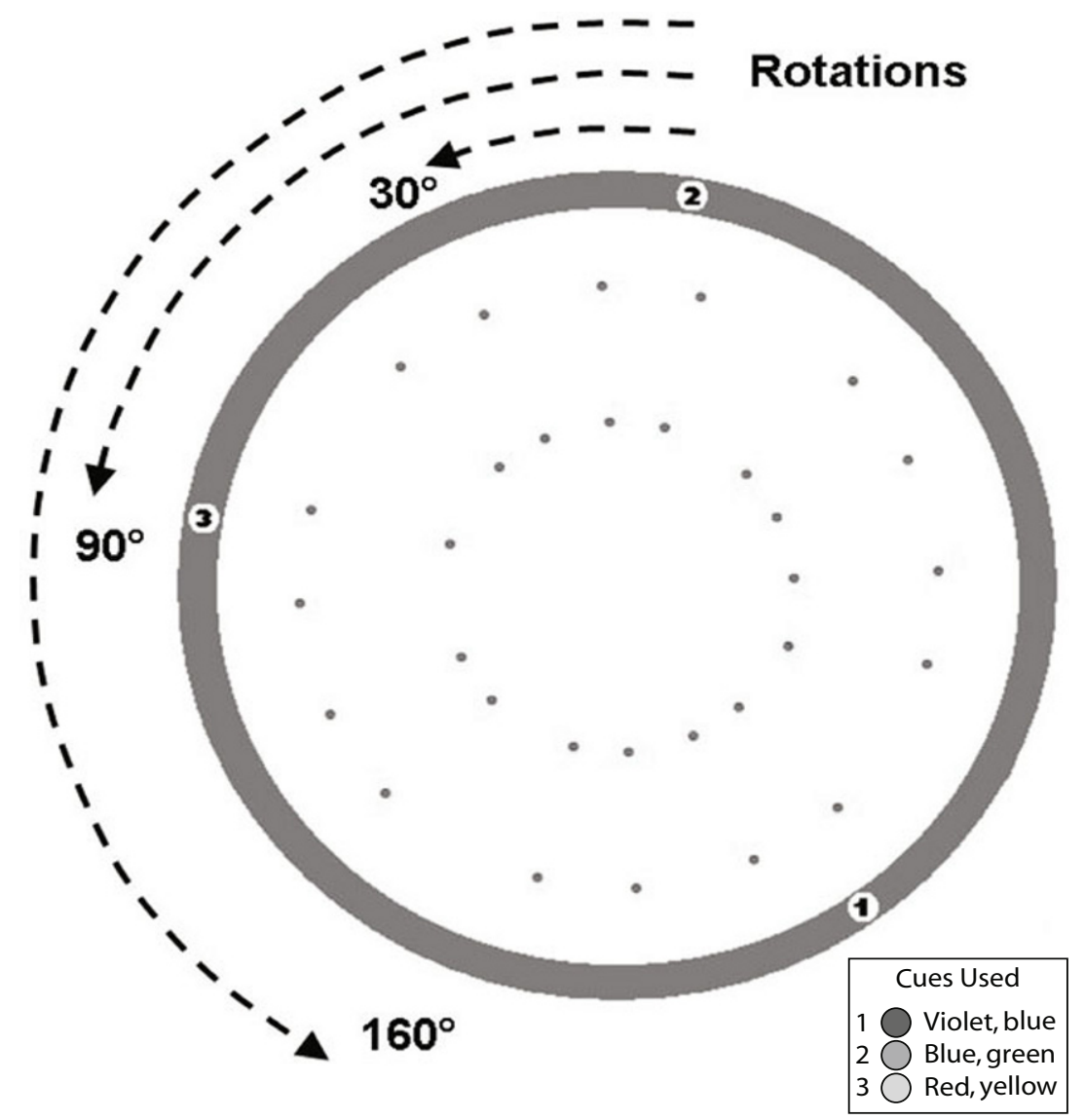

Figure 3. Circular task field of radius of 212 pixels, with 32 target dot locations distributed over the circular area (16 dots located at a radius of 92 pixels and 16 located at a radius of 168 pixels). Within each of four quadrants, dots were located at one of four different angles $\left(3^{\circ}, 25^{\circ}, 43^{\circ}\right.$, and $\left.75^{\circ}\right)$. A black border 20 pixels in width defined the circle. Numbers refer to cue locations at $305^{\circ}, \mathbf{8 0}^{\circ}$, and $170^{\circ}$. Reference cues were small circles presented in two different colors as indicated. The three different degrees of rotation used in Experiment 2 are illustrated by arced arrows, representing how far the cues would rotate on the response trial $\left(30^{\circ}, 90^{\circ}\right.$, or $\left.160^{\circ}\right)$. The size of the dots does not correspond to the actual scale of presentation.

in order to provide sufficient data for modeling the predicted biases. Sixteen dots were located at a radius of 92 pixels (short radius from the center), and the other 16 dots were located at a radius of 168 pixels (long radius from the center). In each of these two sets, four different angles $\left(3^{\circ}, 25^{\circ}, 43^{\circ}\right.$, and $\left.75^{\circ}\right)$ were used and presented in each of the four quadrants inferred from horizontal and vertical boundaries implied by a cue-independent frame of reference. Dot values were presented successively in random order, and the circular field was centered on the screen. We manipulated context between participants by including different numbers of external reference cues, with no external reference cue along the circular region $(n=$ $20)$, one external reference cue $(n=22)$, or three external reference cues $(n=20)$. Participants were randomly assigned to one of the three conditions.

Three dependent variables were recorded to examine spatial memory. Absolute error was measured in pixels as an absolute distance from the actual stimulus value to the remembered dot location, to assess overall accuracy of place memory. Angular bias was measured in degrees by subtracting the angle of the actual location from the angle of the reproduced location. A negative value indicated a clockwise bias, whereas a positive value indicated a counterclockwise angular bias. Radial bias was measured in pixels by subtracting the radial distance of the actual point from the radial distance of the observed point. A negative radial value indicated a radial bias toward the center of the circle, whereas a positive value indicated a radial bias toward the circumference of the circle.

\section{Materials and Apparatus}

All materials and instructions were presented on IBM-compatible computers with 15 -in. $(38-\mathrm{cm})$ monitors. The circular region was identical to the white background and was separated by a 20-pixel black circle in video graphics array mode at a resolution of $640 \times$ 480 pixels with a radius of 212 pixels. Note that the quadrants were not explicitly represented. A red dot, 5 pixels in diameter, was presented within the circular figure in each trial. No dot position was repeated in the stimulus set. Depending on the condition, one, three, or no external reference cues were located along the circular region. In the one-cue condition, the reference cue (blue and violet in color) was located at $305^{\circ}$ along the circle. In the three-cue condition, the reference cues were located at $80^{\circ}$ (blue and green in color), $170^{\circ}$ (red and yellow in color), and $305^{\circ}$ (blue and violet in color) along the circle (see Figure 3). The colors for the cues were chosen to make them highly distinguishable from each other. The locations of the cues were designed to create markedly different patterns of bias for cases in which the cues were used as prototypes, as opposed to the use of the standard four-quadrant representation. 
Table 1

Degrees of Freedom $(d f s)$ and $F$ Values for 3 (Reference Cues) $\times$ 2 (Radius) $\times 4$ (Angle) $\times 4$ (Quadrant) Mixed Factorial ANOVAs of Experiment 1

\begin{tabular}{|c|c|c|c|c|}
\hline Source & $d f$ & $\begin{array}{c}\text { Angular } \\
\text { Bias }\end{array}$ & $\begin{array}{c}\text { Absolute } \\
\text { Error }\end{array}$ & $\begin{array}{c}\text { Radial } \\
\text { Bias }\end{array}$ \\
\hline Cue condition (C) & $(2,59)$ & 0.12 & 2.57 & 0.78 \\
\hline Radius (R) & $(1,59)$ & 2.90 & $40.95^{* * *}$ & $97.97^{* * *}$ \\
\hline $\mathrm{R} \times \mathrm{C}$ & $(2,59)$ & 0.40 & 2.33 & 0.51 \\
\hline Quadrant (Q) & $(3,177)$ & $10.55^{* * *}$ & 1.42 & $16.92^{* * *}$ \\
\hline $\mathrm{Q} \times \mathrm{C}$ & $(6,177)$ & 2.95 & 0.90 & 1.66 \\
\hline Angle (A) & $(3,177)$ & $28.21^{* * *}$ & 2.31 & $5.34^{* *}$ \\
\hline $\mathrm{A} \times \mathrm{C}$ & $(6,177)$ & 0.56 & 1.42 & 1.08 \\
\hline $\mathrm{R} \times \mathrm{Q}$ & $(3,177)$ & $5.80^{* *}$ & $5.20^{* *}$ & $5.04^{* *}$ \\
\hline $\mathrm{R} \times \mathrm{Q} \times \mathrm{C}$ & $(6,177)$ & 0.98 & 1.39 & 1.78 \\
\hline $\mathrm{R} \times \mathrm{A}$ & $(3,177)$ & 2.07 & 4.04 & 0.06 \\
\hline $\mathrm{R} \times \mathrm{A} \times \mathrm{C}$ & $(6,177)$ & 1.17 & 0.73 & 0.94 \\
\hline $\mathrm{Q} \times \mathrm{A}$ & $(9,531)$ & $3.56^{* *}$ & $4.05^{* * *}$ & 2.51 \\
\hline $\mathrm{Q} \times \mathrm{A} \times \mathrm{C}$ & $(18,531)$ & 1.29 & 1.00 & 0.50 \\
\hline $\mathrm{R} \times \mathrm{Q} \times \mathrm{A}$ & $(9,531)$ & 2.09 & 1.07 & 2.14 \\
\hline $\mathrm{R} \times \mathrm{Q} \times \mathrm{A} \times \mathrm{C}$ & $(18,531)$ & 0.93 & 0.96 & 1.08 \\
\hline
\end{tabular}

\section{Procedure}

Groups of 1-5 participants were tested at the same time within a laboratory room with computer terminals spaced approximately $1 \mathrm{~m}$ apart. Participants were first presented with the general instructions and were told that the experiment concerned spatial memory and estimating point locations. After reading the general instructions, participants were presented two learning trials with feedback and one learning set including five trials without feedback. This was followed by the actual test with 32 different dot locations. Each dot was on screen for $1 \mathrm{sec}$, then covered by a dynamic checkerboard mask for $1.5 \mathrm{sec}$, followed by a blank circle. The checkerboard mask consisted of white and black $10 \times 10$ pixel quadrants covering the circular region. The colors of the quadrants were exchanged three times after $0.5 \mathrm{sec}$, creating a moving pattern to avoid fixation. A cross appeared at the center of the circle, serving as a marker for the participant to indicate the to-be-remembered dot location on the respond display by using the mouse. Responses were recorded in pixel units of the marked locations.

\section{Results}

\section{Data Analyses}

The observed dot locations were used to generate the dependent variables in all analyses. Because a blatant misremembering of a location can obscure systematic effects, we developed methods for eliminating these data points. An observed value was designated an outlier for all three dependent variables on the basis of absolute error and angular bias. For absolute error, more than two SDs from the mean of estimates for that dot location across all participants within the corresponding cue condition indicated that the observation was an outlier. For angular bias, a deviation of more than $90^{\circ}$ in either direction was an outlier, because deviations of this magnitude were likely due to gross errors of memory. Combining both methods, these blatantly misremembered locations were recorded and replaced by the mean of the remaining values for the specific dot location within each of the three conditions. The number of data points replaced was as follows: 12 out of 640 in the zero-cue condition, 13 out of 704 in the one-cue condition, and 11 out of 640 in the three-cue condition (with roughly equal numbers replaced for short and long radii). Because replacing missing data with means increases the Type I error rate, the lower value of $\alpha=.01$ was used to determine significance for all statistical tests. Violations of compound symmetry were addressed via the use of the Greenhouse-Geisser degrees of freedom correction factor (Greenhouse \& Geisser, 1959).

\section{Angular Bias}

Two predictions were made for angular bias: (1) We hypothesized that cue condition would have no impact on human spatial memory in a static task field; (2) we hypothesized the estimation process would rely on four quadrant categories resulting from the imposition of implicit horizontal and vertical boundaries and demonstrated by a single zero-bias point within each of these quadrants that corresponded to the prototype according to the category-adjustment model.

A 3 (cue condition) $\times 2$ (radius) $\times 4$ (angle) $\times 4$ (quadrant) mixed factorial ANOVA was used to analyze these angular bias data, with Table 1 summarizing the results in the corresponding column. No interactions involving reference cues were statistically significant. This result supports the cue-independent representation, in that angular bias in estimation was unaffected by manipulation of number of cues. Consistent with a category-adjustment model, a significant main effect for angle was noted, supporting the predictions of the original and modified versions. A significant quadrant $\times$ angle interaction indicated that effects of angle were moderated by quadrant. Furthermore, a significant radius $\times$ quadrant interaction was noted, with an increase in angular bias for the short radius in Quadrants 2 and 3, compared with the long radius.

Because the different cue conditions did not significantly differ, the angular bias scores were combined across all three conditions and fit to the category-adjustment model and the fuzzy-boundary model. The Huttenlocher et al. (1991) category-adjustment model predicts a significant angle effect with a linear trend and consists of five fitted parameters, the weighting of fine-grain memory $(\lambda)$, and the four prototypes $\left(p_{1}, p_{2}, p_{3}, p_{4}\right)$. The model provided a moderately good fit of the bias effects in the estimation data on the basis of proportion of variance explained $\left(R^{2}=.52\right)$. Table 2 presents the estimated parameter values, and Figure 4A illustrates the model fit. A test of the model fit to the empirical data was determined by conducting an ANOVA on the residuals of the model. Results revealed that the significant angle effect and the angle $\times$ quadrant and quadrant $\times$ radius interactions could not be fully explained by this simple version of the category-adjustment model, because these effects remained significant.

We next fit the fixed-quadrants fuzzy-boundary model of Equation 3. This model differs from the basic model in that it takes uncertainty of categorical classification into account. Different versions of this model can be generated by freeing different parameter values. To fit the set of 32 target means to the models, we used the iterative nonlinear regression procedure within SYSTAT (Wilkinson, 1989) with a least squared error criterion and the Gauss-Newton method of steepest descent. The model fit was analyzed using two criteria: (1) Parameters were constrained as long 
Table 2

Parameter Values and Fit Indices for Models of Angular Bias (Experiment 1)

\begin{tabular}{|c|c|c|c|c|c|c|c|c|}
\hline \multirow[b]{2}{*}{ Model/Radii } & \multicolumn{6}{|c|}{ Parameter } & \multirow[b]{2}{*}{$R^{2}$} & \multirow{2}{*}{$\begin{array}{c}\text { Remaining } \\
\text { Significan } \\
\text { Effects }\end{array}$} \\
\hline & $p_{1}$ & $p_{2}$ & $p_{3}$ & $p_{4}$ & $\lambda$ & $c$ & & \\
\hline \multicolumn{9}{|l|}{5 Parameters } \\
\hline Short & $44.84^{\circ}$ & $177.97^{\circ}$ & $227.81^{\circ}$ & $291.99^{\circ}$ & .974 & - & .517 & 3 \\
\hline Long & $44.84^{\circ}$ & $177.97^{\circ}$ & $227.81^{\circ}$ & $291.99^{\circ}$ & .974 & - & & \\
\hline \multicolumn{9}{|l|}{6 Parameters } \\
\hline Short & $48.17^{\circ}$ & $157.04^{\circ}$ & $224.66^{\circ}$ & $224.66^{\circ}$ & .929 & 0.059 & .737 & 1 \\
\hline Long & $48.17^{\circ}$ & $157.04^{\circ}$ & $224.66^{\circ}$ & $224.66^{\circ}$ & .929 & 0.059 & & \\
\hline \multicolumn{9}{|l|}{10 Parameters } \\
\hline Short & $43.73^{\circ}$ & $170.80^{\circ}$ & $218.95^{\circ}$ & $310.39^{\circ}$ & .931 & 0.062 & .849 & 0 \\
\hline Long & $52.90^{\circ}$ & $144.55^{\circ}$ & $230.47^{\circ}$ & $300.35^{\circ}$ & & & & \\
\hline
\end{tabular}

Note-The 5-parameter model is the basic category-adjustment model; the 6- and 10-parameter models are versions of the fixed-quadrants fuzzy-boundary model. $p$, prototype value; $\lambda$, weight of fine-grain memory; $c$, sensitivity parameter.

as the result was not a significant drop in $R^{2} ;(2)$ the values predicted by the proposed models were subtracted from the empirical values, and an ANOVA was conducted on these residuals to determine the remaining significant effects not explained by the model. For the fixed-quadrants fuzzy-boundary model, the fit was conducted simultaneously, starting with 12 parameters free to vary. The final fuzzy-boundary model presents two versions with either 6 or 10 free parameters. The 6-parameter fuzzy-boundary model fits $\lambda, c$, and four prototype values, with no parameters varying across radius. In comparison with the 5-parameter category-adjustment model, it provided a very good fit of the bias variance in the estimation data $\left(R^{2}=.74\right)$. Table 2 presents the estimated parameter values, and Figure 4B illustrates the 6-parameter model fit. An ANOVA conducted on the residuals of the model confirmed the superiority of the 6-parameter fuzzy-boundary model relative to the 5-parameter category-adjustment model, with no significant angle effect or angle $\times$ quadrant interaction remaining. However, the radius $\times$ quadrant interaction remained significant.

A more complex version of the fixed-quadrants fuzzyboundary model was constructed to explain the two-way interaction effect. The 10-parameter fuzzy-boundary model fit $\lambda$ and $c$ along with four different prototype values to each of the two radii separately. Table 2 presents the estimated parameter values, and Figure $4 \mathrm{C}$ illustrates the 10-parameter model fit. The model provided a better fit to the data $\left(R^{2}=.85\right)$. An ANOVA conducted on the residuals showed no significant effects remaining.

In summary, the 5-parameter category-adjustment model did not fully explain the angle effect and two interactions, whereas the angle effect and one of the interactions were explained by the 6-parameter fuzzy-boundary model. The 10-parameter fixed-quadrants fuzzy-boundary model that postulated slightly different prototypes being used for the different radius conditions accounted for all significant effects in the data. Finally, we noted that although the estimated prototype locations were generally close to the midpoints of the quadrants $\left(45^{\circ}, 135^{\circ}, 225^{\circ}\right.$, and $315^{\circ}$ ), a notable exception was the location estimated for Prototype 2. However, such deviations are fairly common. For example, in Huttenlocher et al.'s (1991) Experiment 2, the Quadrant 1 prototype was estimated to be close to $73^{\circ}$. Thus, substantial variation in estimates is possible across experiments and procedures.
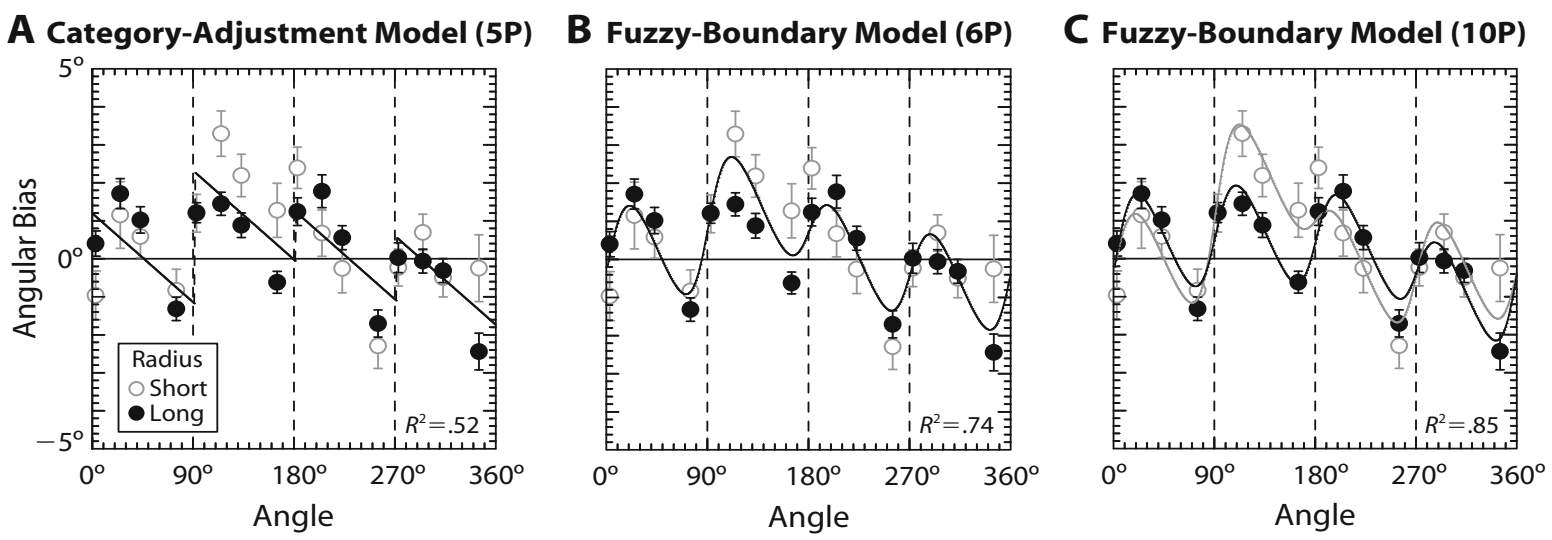

Figure 4. Fit to mean signed angular bias (Experiment 1). The error bars indicate the standard error of the mean. Functions indicate model predictions. (A) 5-parameter category-adjustment model. (B) 6-parameter fixed-quadrants fuzzy-boundary model. (C) 10-parameter fixed-quadrants fuzzy-boundary model. 


\section{Absolute Error}

A parallel $3 \times 2 \times 4 \times 4$ mixed factorial ANOVA was used to analyze absolute error data, with Table 1 summarizing the results in the corresponding column. No interactions involving the number of cues were statistically significant. This result again supports the cue-independent representation, because there is no evidence that number of available cues affected absolute error in estimation. There was a significant radius effect on absolute error, with reduced accuracy for the short radius $(M=11.97$, $S D=1.56)$, as compared with the long radius $(M=9.58$, $S D=1.30)$. This effect was moderated by an interaction with quadrant. This finding indicated an increased absolute error for the short radius in Quadrants 2 and 3, as compared with the long radius. The significant quadrant $X$ angle interaction indicates the angle dependency of absolute error in each of the four quadrants. The observed significant effects are not particularly relevant to our model assessment and are not particularly large, so we will not analyze them further.

\section{Radial Bias}

A parallel $3 \times 2 \times 4 \times 4$ mixed factorial ANOVA was conducted on the measures of radial bias, with results shown in Table 1 in the corresponding column. Once again, no interactions involving the number of cues were statistically significant. This result again supports the cueindependent representation, because there is no evidence that number of available cues affected radial estimation. The significant main effect of radius indicated that estimates were driven toward the circumference $(+)$ for the short radius $(M=6.08, S D=2.50)$ and nearly no bias occurred for the long radius $(M=0.49, S D=1.90)$. The effect was moderated by quadrant, indicating an increased radial bias for the short radius in Quadrants 2 and 3, as compared with the long radius. The significant angle effect indicated that the radial bias was smallest for $3^{\circ}$, thus decreasing for angles near the inferred boundary region. In general, the main effect of radius was consistent with the Huttenlocher et al. (1991) category-adjustment model, in the sense that the inferred radial prototype was closer to the value of the longer radius.

\section{Discussion}

In Experiment 1, we were interested in the impact of external reference cues on spatial memory when locating stimuli in a static task field. Results indicated that when the spatial reference field remains fixed, spatial memory is largely independent of available external cues, supporting the use of a cue-independent frame of reference. These bias effects were best described by the fixed-quadrants fuzzy-boundary model, a modification of the basic category-adjustment model similar to the uncertain-boundary model of Huttenlocher et al. (1991). The clear reduction of bias near the boundaries supported the fuzzy-boundary and uncertain-boundary versions of the category adjustment model over the basic model, which fit significantly worse. This reduction can be attributed to the tendency to recruit more than one prototype for targets located near a boundary.
The cue independence of spatial encoding within a static response field was supported by a lack of any significant interactions with cue condition in the three ANOVAs. These results suggest that reference cues were largely ignored by participants when making spatial estimates. Instead, they appeared to rely on the categories or quadrants determined by $0^{\circ}, 90^{\circ}, 180^{\circ}$, and $270^{\circ}$ boundaries, with the prototypes located near the middle of each quadrant. One reason for the lack of cue effects may be that the cue-independent representation was already providing highly accurate estimates. As can be seen in Figure 4, even the most biased estimates were within a few pixels of the actual location. From a broader rational memory perspective, and given the highly adaptive nature of the cue-independent frame of reference, participants had no need to integrate cues into their spatial representations, since there would be little if any additional benefit.

Naturally, a problem in drawing conclusions on the basis of retaining the null hypothesis relates to the power of the experiment to detect the predicted changes. A key to calculating power is to estimate effect size. If we use the theoretical predictions illustrated in Figures 1 and 2 as baseline measures of effect size, the power would be well above .99; indeed, these predicted effects are so visually dramatic that one would not really need a statistical test to confirm their occurrence. Although cue was manipulated between subjects, the key comparisons that would indicate cue effects were all attributable to the interaction of cue with a withinsubjects factor, such as angle, quadrant, or radius. The use of these within-subjects error terms greatly enhanced the power of the experiment to detect cue effects. We will return to this issue after reporting the results of Experiment 2, since those results will provide a clearer basis for calculating the power of Experiment 1 to detect cue-based effects.

Experiment 1 also shed some light on the adequacies of the simple version of the category-adjustment model. The model fits to the angular bias scores were evaluated on the basis of two criteria: (1) proportion of systematic variance explained and (2) elimination of significant effects when residuals were analyzed. Both criteria suggested that estimates could best be described by the fixed-quadrants fuzzyboundary model. The two criteria used to evaluate the models indicated (1) that this model explained more of the variance than did the simple category-adjustment model; and (2) that only one effect could not be explained by the 6-parameter version of the model, in contrast to three unexplained significant effects by the basic category-adjustment model. The significant quadrant $\times$ radius interaction indicated changes in the prototypes for radii that could be explained only by the more complex 10-parameter model. These results suggest that radius is implicated in the prototype recruitment process when spatial location in a circular region is estimated. Note that these effects of radius on prototypes may not be large, but small changes in relative spacing of prototypes can result in significant interactions of radius and quadrant.

Interestingly, a significant interaction between radius and quadrant was found for radial bias, indicating a robust effect in terms of overshooting the value of the short radius on the left side of the display. This finding suggests a leftward bias in either the representation or the motor 
responses associated with the short radius. It should be noted that in all three conditions, the error and bias effects were very small in magnitude but highly significant and robust, nevertheless.

In conclusion, results from Experiment 1 speak to our fundamental research question of whether external cues would be incorporated into the spatial representation and used in the estimation process for fixed spatial displays. The clear answer to this question is that spatial memory under these circumstances is not influenced by peripheral reference cues, a finding that indicated use of a cue-independent frame of reference. Whether this cue-independent frame of reference is based on intrinsic axes, as suggested by Huttenlocher et al. (1991), or on a viewer-based frame of reference (Wedell et al., 2007) cannot be answered with this experiment. We next investigated whether this finding could be generalized to dynamic response fields.

\section{EXPERIMENT 2}

Experiment 1 showed that in a spatial memory task with a static task field, participants simply ignored the available peripheral cues and based their spatial categories on spontaneously imposed fixed horizontal and vertical boundaries. Experiment 2 increased the potential for cue use by creating a more dynamic task field setting. The dynamism of the task field was introduced by including trials in which the task field was rotated to different degrees, thus destabilizing the participants' orientation to the task field. The model development described earlier showed how the category-adjustment model can be modified to account for fuzzy boundaries and cue-based prototypes in the basic dot location task. The patterns of bias when cues were used to determine prototype locations were demonstrated to be quite different, depending on the number and location of cues (see Figure 2).

We hypothesized that a dynamic task field would render the fixed horizontal and vertical boundaries typically used in the location task fairly irrelevant. We conjectured that introducing an orientation shift with the rotation trials would destabilize the participants' orientation, so that cues would have to be used in order to maintain orientation, and thus the vertical and horizontal axes would be disregarded by participants. Categorical coding of spatial locations would therefore be determined by the available peripheral cues. Our specific model of this process is that these cues would serve as prototypes defining categories that would be integrated with fine-grain information to produce estimates of spatial location. We hypothesized that these effects should be present on the nonrotation trials as well, because it would be difficult to maintain dual spatial encoding (one cue based and the other quadrant based).

Figure 3 illustrates key aspects of the experimental setup, with one or three cues appearing on the periphery of the circle in the orientation shown. After the checkerboard mask obscured the target's location, the orientation either remained the same or shifted by $30^{\circ}, 90^{\circ}$, or $160^{\circ}$. The shift in orientation of the task field was indicated by the shifting of the cues. For example, when the display was rotated $160^{\circ}$, the cue marked 2 in Figure 3 would rotate to the position indicated by the corresponding arrowhead near the bottom left of the display, with the other cues rotating in a like manner. Participants were instructed to indicate the location of the target in the rotated task field.

In addition to predicting changes in the pattern of bias, we also hypothesized that cue condition would affect the accuracy of human spatial memory in terms of degree of absolute error. The cue-based fuzzy-boundary model predicts that absolute error should decrease when more peripheral cues are available, because bias represents one component of absolute error. As is shown in Figure 2, bias is much greater in the one-cue condition than in the three-cue condition, because bias increases as the distance to the nearest prototype increases. By including more prototypes, the magnitude of bias is reduced and hence absolute error is reduced (assuming that all other sources of error remain constant). Apart from our model-based prediction of reduced absolute error with more cues, the literature supports the reduction of error near cues (Cook \& Tauro, 1999; Kamil \& Cheng, 2001; Werner \& Diedrichsen, 2002). In our study we focused on external or peripheral cues surrounding the task field and examined whether or not proximity to an available cue is related to accuracy in humans; we then hypothesized that absolute error would be reduced near available peripheral cues when these were used as prototypes.

Finally, for radial bias, we hypothesized that, as in Experiment 1, participants would be biased toward the circumference for the short radius and to the opposite direction (toward the center) for the long radius. Our cue-based fuzzy-boundary model does not predict any effects of cues on radial bias. However, findings of Werner and Diedrichsen (2002) and Schmidt, Werner, and Diedrichsen (2003) suggest that landmarks may systematically distort estimates of targets in close proximity to those landmarks. Consistent with these findings, we speculated that a radial bias toward the external cues might occur for targets nearest the cues (a quadrant $\times$ radius interaction).

Finally, note that although Experiment 2 provided data for nonrotation and rotation trials, our analyses focused only on the nonrotation trials. As will be discussed in the Results section, our focus on the nonrotation trials arose from three concerns. First, the nonrotation trials presented the clearest comparison with the results from Experiment 1, since the procedure for these trials was exactly the same across the two experiments. Second, there was the problem that large numbers of misremembered points were designated as outliers in the rotation conditions, making valid statistical analyses difficult. Because most of these outliers were simply gross memory errors, they could not be used to reveal any significant pattern related to the frame of reference being used by participants. Third, we did not believe that the added complexities in modeling the results obtained from these trials were directly relevant to the hypotheses being tested.

\section{Method}

\section{Participants and Task}

Fifty-four undergraduate students from the University of South Carolina psychology department participant pool took part in the experiment in exchange for course credits. All participants attempted to 
reproduce the same locations of 32 dots in a circular region as presented in Experiment 1. The apparatus and materials were the same as those in Experiment 1. Dot values were again presented successively in random order, and the circular field was centered on the screen. In order to create a dynamic task field, the response task field was rotated $0^{\circ}, 30^{\circ}, 90^{\circ}$, and $160^{\circ}$ after the checkerboard mask. Furthermore, all participants were tested under two cue conditions. In the one-cue condition, an external cue appeared at $305^{\circ}$ along the perimeter of the circle. In the three-cue condition, unique peripheral cues appeared at $80^{\circ}, 170^{\circ}$, and $305^{\circ}$ on the perimeter. Thus, each cue set consisted of 128 trials (four rotations of 32 dot locations). Note that rotation consisted of moving the locations of the peripheral cues around the field by the prescribed angle. Figure 3 provides a schematic illustration of the different angles of rotation, achieved by rotating the display that included the cues through the specified angle. A between-subjects variable was the order of presentation of sets (one cue first followed by the three-cue condition or three cues first followed by the one-cue condition). Participants were randomly assigned to the between-subjects conditions ( $n=27$ for both). As in Experiment 1, the three dependent variables were absolute error, angular bias, and radial bias.

\section{Procedure}

As in Experiment 1, groups of 1-5 participants were tested at the same time within a laboratory room with computer terminals spaced approximately $1 \mathrm{~m}$ apart. After reading the general instructions, participants were presented with two learning trials with no rotations, followed by specific instructions about the rotation process, as follows:

In the next part of the experiment, the point will appear, the checkerboard pattern will appear, and the circle will rotate to a new orientation. Pay attention to the rotation. Rotating the circle changes the location of the point within the circle. Your task is to indicate the point's location after the circle is rotated by using the mouse.

After these specific instructions, two learning trials for the rotation trials were given, with feedback, followed by one learning set consisting of five trials, without feedback. These three learning sets were followed by this introduction to the actual test sets:

Now the first set of testing trials start. The point will appear, the checkboard pattern will appear, and the circle will rotate to a new orientation. Notice that in some trials the circle stays the same and does not rotate. Use the mouse to indicate the point's location.

After these instructions, two actual test sets were presented with 128 different dot locations. The timing and procedure for responding were the same as in Experiment 1. After the first set, there was a 3-min break. In the second set, the cue condition was changed and participants rated the same dot locations presented successively in random order. Responses by the participant were recorded in pixel units of the marked locations.

\section{Results}

The observed dot locations were used to generate the dependent variables in all analyses. The method for eliminating outliers was the same as in Experiment 1. The data points that represented blatant misremembering of locations were defined separately for each of the field rotations and for each of the cue conditions in the order in which they appeared. These data points were recorded and replaced by the mean of the remaining values for the specific point in the corresponding condition.

As was described in the introduction, our data analyses in Experiment 2 focused only on the nonrotation trials, for reasons of comparability with Experiment 1, number of outliers, and increased complexity in modeling. Here, we simply report the number of outlying data points in the rotation trials. For rotations of $30^{\circ}, 90^{\circ}$, and $160^{\circ}$, the proportion of outlying points were $5.32 \%, 13.34 \%$, and $22.60 \%$, respectively. An ANOVA conducted on number of outlying points for each participant revealed significant differences between the nonrotation and rotation trials. In contrast, the number of data points that were replaced for the nonrotation trials was $3.33 \%$. Specifically, out of 432 data points, the number of outliers was as follows: one cue first, short $=22$, long $=15$; one cue second, short $=13$, long $=11$; three cues first, short $=14$, long $=12$; three cues second, short $=16$, long $=12$. Analyses conducted on the nonrotation trials examined the estimation process unconfounded by rotation bias. These nonrotation trials afford the use of both strategies - cue-independent or cuebased frame of reference - in order to locate the dot in the circular region and so constitute a strong test of the effects of field dynamics. Given the large number of outliers and our focus on the directly comparable conditions in Experiments 1 and 2, we will not report results for the rotation trials in this article. For the same reasons as those stated in Experiment 1, the Type I error rate was lowered to $\alpha=.01$ to determine significance for all statistical tests, accounting for reduced variability introduced by substitution with means.

\section{Angular Bias}

We hypothesized that different patterns would be observed for one- and three-cue conditions, represented by interactions with cue condition. A 2 (cue condition) $\times$ 2 (radius) $\times 4$ (angle) $\times 4$ (quadrant) mixed factorial ANOVA on angular bias was conducted, with Table 3 summarizing the results in the column labeled Angular Bias. A significant cue effect was noted, with a decrease in positive bias in the three-cue condition relative to the one-cue condition. More relevant to the cue-dependent memory hypothesis, cue condition significantly interacted with quadrant, with angle, with radius $\times$ quadrant, and with quadrant $\times$ angle. In addition, significant effects were noted for quadrant, angle, quadrant $x$ angle, and radius $\times$ quadrant $\times$ angle. The key finding, as is shown in Table 3, was that cue was involved in four interaction effects. These significant cue interactions with angular bias prompted us to conduct separate analyses for one-cue and three-cue conditions to better describe these.

In the one-cue condition, a 2 (order: first or second) $\times$ 2 (radius) $\times 4$ (angle) $\times 4$ (quadrant) mixed factorial ANOVA on angular bias was conducted and revealed no significant order effect or order interaction. Results of the ANOVA are shown in the column designated Angular Bias in Table 4.

Because of a lack of significant order effects, the mean angular bias scores were combined across orders and were fit by the fuzzy-boundary model. Different versions of the cue-based fuzzy-boundary model were generated by freeing parameter values and using the same iterative nonlinear regression procedure as in Experiment 1. The model fit was analyzed using the same two criteria as in Experiment 1 . The fit of the fuzzy-boundary model to the one- 
Table 3

Degrees of Freedom ( $d f s$ ) and $F$ Values for 2 (Reference Cues) $\times$ 2 (Radius) $\times 4$ (Angle) $\times 4$ (Quadrant) Within Factorial ANOVAs of Experiment 2 in the Nonrotation Condition

\begin{tabular}{lcccc}
\hline \multicolumn{1}{c}{ Source } & Angular & Absolute & Radial \\
Cue condition $(\mathrm{C})$ & $(1,53)$ & $7.11^{* *}$ & $29.95^{* * *}$ & 2.03 \\
Radius $(\mathrm{R})$ & $(1,53)$ & 3.42 & $15.53^{* * *}$ & $240.42^{* * *}$ \\
$\mathrm{R} \times \mathrm{C}$ & $(1,53)$ & 0.48 & 5.75 & 0.00 \\
Quadrant (Q) & $(3,159)$ & $26.23^{* * *}$ & $21.62^{* * *}$ & $8.20^{* * *}$ \\
$\mathrm{Q} \times \mathrm{C}$ & $(3,159)$ & $69.82^{* * *}$ & $9.84^{* * *}$ & $17.26^{* * *}$ \\
Angle $(\mathrm{A})$ & $(3,159)$ & $6.60^{* * *}$ & 2.27 & 0.39 \\
$\mathrm{~A} \times \mathrm{C}$ & $(3,159)$ & $5.53^{* * *}$ & 2.45 & 1.88 \\
$\mathrm{R} \times \mathrm{Q}$ & $(3,159)$ & 3.53 & 3.33 & 1.09 \\
$\mathrm{R} \times \mathrm{Q} \times \mathrm{C}$ & $(3,159)$ & $10.67^{* * *}$ & 0.99 & 1.69 \\
$\mathrm{R} \times \mathrm{A}$ & $(3,159)$ & 3.76 & 0.79 & 1.07 \\
$\mathrm{R} \times \mathrm{A} \times \mathrm{C}$ & $(3,159)$ & 1.25 & 0.99 & 0.60 \\
$\mathrm{Q} \times \mathrm{A}$ & $(9,477)$ & $5.30^{* * *}$ & $5.04^{* * *}$ & $2.85^{* *}$ \\
$\mathrm{Q} \times \mathrm{A} \times \mathrm{C}$ & $(9,477)$ & $11.88^{* * *}$ & $2.85^{* *}$ & $5.93^{* * *}$ \\
$\mathrm{R} \times \mathrm{Q} \times \mathrm{A}$ & $(9,477)$ & $3.93^{* * *}$ & $3.69^{* * *}$ & 1.70 \\
$\mathrm{R} \times \mathrm{Q} \times \mathrm{A} \times \mathrm{C}$ & $(9,477)$ & 0.93 & 2.06 & $3.41^{* * *}$ \\
\hline$* * p<.01$. & $* * * p<.001$. & & &
\end{tabular}

cue condition began with 6 parameters free to vary. We present two versions of the final fuzzy-boundary model with 3 or 4 free parameters, respectively. The 3-parameter fuzzy-boundary model fit $\lambda, c$, and one prototype value $p$, with no parameters varying with radius. It provided a very good fit of the bias variance in the estimation data $\left(R^{2}=.91\right)$. The row designated " 3 parameters" in Table 5 presents the estimated parameter values.

However, a 2 (radius) $\times 4$ (angle) $\times 4$ (quadrant) within factorial ANOVA on the residuals from this model revealed a quadrant $\times$ radius interaction effect $[F(3,159)=$ $\left.5.74, p_{\mathrm{GG}}=.001\right]$. No other significant effect was noted. In order to overcome this weakness in explaining the significant interaction effect, a more complex model was fit. The 4-parameter cue-based fuzzy-boundary model addressed this problem, with $\lambda_{1}, \lambda_{2}, c$, and one prototype value $p\left(R^{2}=.93\right)$ (see the row designated " 4 parameters" in Table 5). An ANOVA on the residuals from this model revealed no significant effects. Figure 5A illustrates the model fit for the 4-parameter model.

In the three-cue condition, a parallel $2 \times 2 \times 4 \times 4$ mixed factorial ANOVA on angular bias was conducted, with results summarized in the Angular Bias column of Table 6. The two significant order interactions led us to fit the mean bias scores for each order condition separately.

Three cues first. The fit was conducted simultaneously, starting with 10 parameters free to vary. The final cue-based fuzzy-boundary model is presented in two versions, with either 5 or 7 free parameters. The 5 -parameter model had $\lambda, c$, and three prototype values $\left(p_{1}, p_{2}, p_{3}\right)$ free to vary and no parameters varying with radius. It provided a reasonable fit of the bias variance in the estimation data $\left(R^{2}=.41\right)$. The row designated " 5 parameters" in Table 7 presents the estimated parameter values.

However, a $2 \times 4 \times 4$ within factorial ANOVA on the residuals from this model revealed a significant angle effect $\left[F(3,78)=5.47, p_{\mathrm{GG}}=.002\right]$ and a significant radius $\times$ angle interaction $\left[F(3,78)=4.88, p_{\mathrm{GG}}=.004\right]$. No other significant effect or interactions were noted. In order to overcome this weakness and explain the significant effects, a more complex model was needed.

Accordingly, a 7-parameter fuzzy-boundary model was developed, with $\lambda_{\mathrm{S}}, \lambda_{\mathrm{L}}, c_{1}, c_{2}$, and three prototype values $\left(p_{1}, p_{2}, p_{3}\right)$ free to vary $\left(R^{2}=.60\right)$ (see the "7 parameters" row in Table 7). An ANOVA on the residuals revealed a significant angle effect $\left[F(3,78)=8.07, p_{\mathrm{GG}}<.001\right]$ as the only significant effect. Figure 5B illustrates the model fit for the 7-parameter fuzzy-boundary model, with the finegrain weighting value varying with radius $\left(\lambda_{\mathrm{S}}\right.$ and $\left.\lambda_{\mathrm{L}}\right)$.

Three cues second. In this condition, the three-cue condition followed the one-cue condition. The pattern of the data suggested that prior estimation in the one-cue condition may have led participants to use fewer than three cues in some of their estimations. Thus, to fit the data, different versions were tested that varied in the number of prototypes used for different radius conditions. The fit was conducted simultaneously, starting with 16 parameters free to vary. The final fuzzy-boundary model presents two versions with either 5 or 6 parameters free to vary. The 5-parameter fuzzy-boundary model had $\lambda, c$, and three prototype values $\left(p_{1}, p_{2}, p_{3}\right)$ free to vary, with no parameters varying with radius. The fit of this model was moderate $\left(R^{2}=.37\right)$. The row designated "5 parameters" in Table 8 presents the estimated parameter values.

A 2 (radius) $\times 4$ (quadrant) $\times 4$ (angle) within factorial ANOVA on the residuals from the 5-parameter model revealed a significant effect of angle $[F(3,78)=9.75$, $\left.p_{\mathrm{GG}}<.001\right]$ and a significant radius $\times$ angle interaction $\left[F(3,78)=6.29, p_{\mathrm{GG}}=.002\right]$. No other significant effects were noted. We then fit a 6-parameter fuzzy-boundary model that consisted of $\lambda, c$, and three prototype values for the long radius $\left(p_{1}, p_{2}\right.$, and $\left.p_{3}\right)$ and only one prototype for the short radius $[p]$. This 6-parameter model provided a better fit $\left(R^{2}=.56\right)$ (see the row designated "6 parameters" in Table 8). An ANOVA on the residuals from this model still revealed a significant angle effect $[F(3,78)=$ $\left.8.20, p_{\mathrm{GG}}<.001\right]$ but no other significant effects or in-

Table 4

Degrees of Freedom ( $d f s$ ) and $F$ Values for 2 (Order) $\times 2$ (Radius) $\times 4$ (Angle) $\times 4$ (Quadrant) Mixed Factorial ANOVAs of Experiment 2 in the Nonrotation Condition for the One-Cue Condition

\begin{tabular}{|c|c|c|c|c|}
\hline Source & $d f$ & $\begin{array}{l}\text { Angular } \\
\text { Bias }\end{array}$ & $\begin{array}{c}\text { Absolute } \\
\text { Error }\end{array}$ & $\begin{array}{c}\text { Radial } \\
\text { Bias }\end{array}$ \\
\hline Order $(\mathrm{O})$ & $(1,52)$ & 0.70 & 3.26 & 1.47 \\
\hline Radius (R) & $(1,52)$ & 4.12 & 1.85 & $168.87^{* * *}$ \\
\hline $\mathrm{R} \times \mathrm{O}$ & $(1,52)$ & 0.27 & 0.31 & 0.37 \\
\hline Quadrant (Q) & $(3,156)$ & $59.43^{* * *}$ & $21.81^{* * *}$ & $19.99^{* * *}$ \\
\hline $\mathrm{Q} \times \mathrm{O}$ & $(3,156)$ & 2.10 & 0.32 & 1.33 \\
\hline Angle (A) & $(3,156)$ & 2.14 & 0.99 & 1.27 \\
\hline $\mathrm{A} \times \mathrm{O}$ & $(3,156)$ & 1.12 & 0.26 & 2.95 \\
\hline $\mathrm{R} \times \mathrm{Q}$ & $(3,156)$ & $5.80^{* *}$ & 3.39 & 0.07 \\
\hline $\mathrm{R} \times \mathrm{Q} \times \mathrm{O}$ & $(3,156)$ & 1.50 & 1.45 & 1.59 \\
\hline $\mathrm{R} \times \mathrm{A}$ & $(3,156)$ & 1.78 & 0.70 & 0.06 \\
\hline $\mathrm{R} \times \mathrm{A} \times \mathrm{O}$ & $(3,156)$ & 0.68 & 0.98 & 2.55 \\
\hline $\mathrm{Q} \times \mathrm{A}$ & $(9,468)$ & $10.05^{* * *}$ & $3.41^{* *}$ & $6.42^{* *}$ \\
\hline $\mathrm{Q} \times \mathrm{A} \times \mathrm{O}$ & $(9,468)$ & 2.12 & 0.84 & 0.61 \\
\hline $\mathrm{R} \times \mathrm{Q} \times \mathrm{A}$ & $(9,468)$ & 1.71 & $2.95^{* *}$ & 2.21 \\
\hline $\mathrm{R} \times \mathrm{Q} \times \mathrm{A} \times \mathrm{O}$ & $(9,468)$ & 1.14 & 1.36 & 0.17 \\
\hline
\end{tabular}


Table 5

Parameter Values and Fit Indices for Models of Angular Bias in the Nonrotation Condition for the One-Cue Condition (Experiment 2)

\begin{tabular}{lccccc}
\hline & \multicolumn{3}{c}{ Parameter } & & $\begin{array}{c}\text { Remaining } \\
\text { Significant } \\
\text { Moffects }\end{array}$ \\
\cline { 2 - 4 } Model/Radii & $p_{1}$ & $\lambda$ & $c$ & $R^{2}$ & 1 \\
\hline 3 Parameters & & & & & \\
$\quad$ Short & $281.625^{\circ}$ & .927 & 0.025 & .908 & 1 \\
$\quad$ Long & $281.625^{\circ}$ & .927 & 0.025 & & \\
4 Parameters & & & & & \\
$\quad$ Short & $281.22^{\circ}$ & .916 & 0.025 & .933 & 0 \\
$\quad$ Long & $281.22^{\circ}$ & .939 & 0.025 & & \\
\hline
\end{tabular}

Note-The 3- and 4-parameter models are versions of the cue-based fuzzy-boundary model. $p_{1}$, prototype value; $\lambda$, weight of fine-grain memory; $c$, sensitivity parameter.

teractions. Figure 5C illustrates the model fit for the 6parameter fuzzy-boundary model.
Absolute Error
The cue-based fuzzy-boundary model implies a decrease in absolute error when more peripheral cues are available (holding $\lambda$ constant). We further predicted a cue interaction with angle and quadrant, on the basis of the idea that ab- solute error is reduced near available peripheral cues when they are used as prototypes (Cook \& Tauro, 1999; Hartley, Trinkler, \& Burgess, 2004; Werner \& Diedrichsen, 2002).
The results of a $2 \times 2 \times 4 \times 4$ mixed factorial ANOVA conducted on these data are summarized in the Absolute Error column of Table 3 . The significant main effect of cue represented decreased absolute error in the three-cue condition $(M=19.66, S D=12.42)$ relative to the one- cue condition $(M=23.80, S D=15.02)$. The significant quadrant effect was moderated by cue condition, resulting in a quadrant $\times$ cue interaction. Furthermore, the signifi- cant quadrant $X$ angle effect was moderated by cue condi- tion, resulting in a three-way interaction. To explicate the interactive effects of cue, we conducted separate ANOVAs on one- and three-cue conditions.

The results of the $2 \times 2 \times 4 \times 4$ mixed factorial ANOVA conducted on the data from the one-cue condition are summarized in the Absolute Error column of Table 4. Paralleling results for angular bias, there was no interaction with the order variable for the one-cue condition. The significant main effect of quadrant represented reduced absolute error in Quadrant 4, consistent with the location of the cue in Quadrant 4. Additionally, quadrant interacted with angle and with angle and radius. This interaction pattern is consistent with our hypothesis that proximity to the cue should lead to reduced error. To test this hypothesis more carefully, we modeled these effects using regression. Absolute error was regressed onto the log transformation of distance from the target to the cue. Figure 6A illustrates the model fit of the absolute error by using the nearest cue location. This two-parameter model (consisting of a slope and intercept) accounted for a large proportion of variance $\left(R^{2}=.59\right)$, indicating that the $\log$ transformation of distance to the cue is a strong predictor of reduction in absolute error in estimation.

The results of the $2 \times 2 \times 4 \times 4$ mixed factorial ANOVA conducted on the data from the three-cue condition are summarized in the Absolute Error column of Table 6. Unlike the ANOVA on angular bias, there was no significant effect or interactions involving order of presentation. The significant main effect of radius indicated a reduction in absolute error of estimation for the more peripheral targets (i.e., long-radius condition). In addition, there was a significant radius $\times$ quadrant $\times$ angle interaction, along with main effects of quadrant and angle, and a quadrant $X$ angle interaction. These effects are all broadly consistent with the hypothesis that proximity to peripheral cues leads to a reduction in absolute error of estimation. To model this effect, we again used a regression analysis, with the predictor variable being the log transformation of distance to the nearest cue. The variance accounted for by the model was high $\left(R^{2}=.60\right)$. Figure 6B illustrates the predicted absolute error from the regression on $\log$ distance to the nearest cue location. In summary, the combined results from one-cue and three-cue conditions indi-
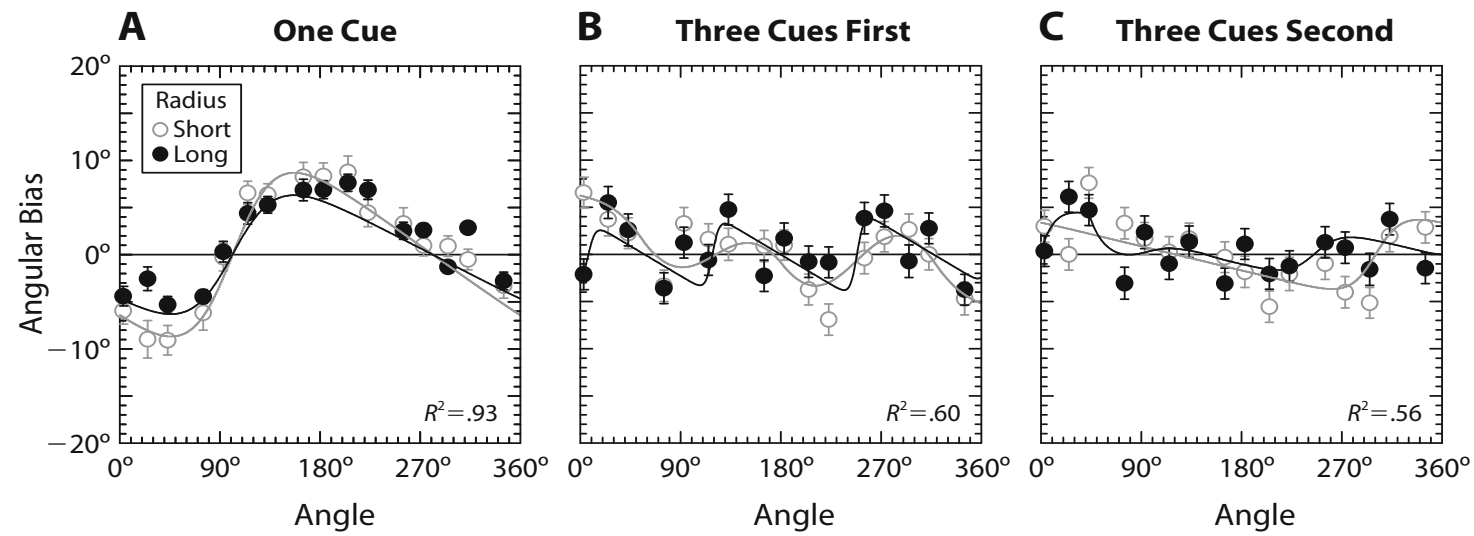

Figure 5. Fit to mean signed angular bias (Experiment 2, nonrotation condition). Error bars represent one standard error, and functions represent the fit of the cue-based fuzzy-boundary model. (A) 4-parameter fit to the one-cue data. (B) 7-parameter fit to the three-cues-first data. (C) 6-parameter fit to the three-cues-second data. 
Table 6

Degrees of Freedom $(d f$ s) and $F$ Values for 2 (Order) $\times$ 2 (Radius) $\times 4$ (Angle) $\times 4$ (Quadrant) Mixed Factorial ANOVAs of Experiment 2 in the Nonrotation Condition for the Three-Cue Condition

\begin{tabular}{llccc}
\hline \multicolumn{1}{c}{ Source } & \multicolumn{1}{c}{$d f$} & $\begin{array}{c}\text { Angular } \\
\text { Bias }\end{array}$ & $\begin{array}{c}\text { Absolute } \\
\text { Error }\end{array}$ & $\begin{array}{c}\text { Radial } \\
\text { Bias }\end{array}$ \\
\hline Order (O) & $(1,52)$ & 0.46 & 3.94 & $9.98^{* *}$ \\
Radius (R) & $(1,52)$ & 0.72 & $25.40^{* * *}$ & $158.74^{* * *}$ \\
$\mathrm{R} \times \mathrm{O}$ & $(1,52)$ & 0.00 & 0.45 & 3.10 \\
Quadrant (Q) & $(3,156)$ & $10.33^{* * *}$ & $7.51^{* * *}$ & 3.14 \\
$\mathrm{Q} \times \mathrm{O}$ & $(3,156)$ & 1.55 & 1.88 & 1.36 \\
Angle $(\mathrm{A})$ & $(3,156)$ & $13.00^{* * *}$ & $4.78^{* *}$ & 0.80 \\
$\mathrm{~A} \times \mathrm{O}$ & $(3,156)$ & $9.02^{* * *}$ & 2.82 & 1.91 \\
$\mathrm{R} \times \mathrm{Q}$ & $(3,156)$ & $8.11^{* * *}$ & 0.49 & 2.80 \\
$\mathrm{R} \times \mathrm{Q} \times \mathrm{O}$ & $(3,156)$ & 0.26 & 0.29 & 0.18 \\
$\mathrm{R} \times \mathrm{A}$ & $(3,156)$ & 3.77 & 1.23 & 1.75 \\
$\mathrm{R} \times \mathrm{A} \times \mathrm{O}$ & $(3,156)$ & $7.47^{* * *}$ & 1.34 & 2.05 \\
$\mathrm{Q} \times \mathrm{A}$ & $(9,468)$ & $6.94^{* * *}$ & $4.71^{* * *}$ & 1.35 \\
$\mathrm{Q} \times \mathrm{A} \times \mathrm{O}$ & $(9,468)$ & 2.49 & 1.05 & 1.28 \\
$\mathrm{R} \times \mathrm{Q} \times \mathrm{A}$ & $(9,468)$ & $3.44^{* *}$ & $2.91^{* *}$ & $2.78^{* *}$ \\
$\mathrm{R} \times \mathrm{Q} \times \mathrm{A} \times \mathrm{O}$ & $(9,468)$ & 0.96 & 0.71 & 0.86 \\
\hline
\end{tabular}

${ }^{* *} p<.01 . \quad{ }^{* * * *} p<.001$.

cate that proximity to a peripheral cue is a good predictor of reduced absolute error in estimation.

\section{Radial Bias}

All the different versions of the category-adjustment model hypothesize a radial bias, typically with the prototypic radius between the short and long radii. We also hypothesized that cue condition could have an impact on prototype recruitment so that there would be an increase in bias toward the cue (cue $\times$ quadrant interaction).

The results of a $2 \times 2 \times 4 \times 4$ mixed factorial ANOVA conducted on these data are summarized in the Radial Bias column of Table 3 . As predicted by the category-adjustment model, the main effect of radius was highly significant. Estimates of short-radius targets were biased toward the circumference, whereas estimates of long-radius targets were biased toward the center. Cue effects were noted in the cue $\times$ quadrant interaction, a cue $\times$ quadrant $\times$ angle interaction, and a cue $\times$ radius $\times$ quadrant $\times$ angle interaction. To tease apart these interactions, we analyzed the different cue conditions separately.

The results of the $2 \times 2 \times 4 \times 4$ mixed factorial ANOVA conducted on the data from the one-cue condition are summarized in the Radial Bias column of Table 4. In addition to the standard main effect of radius, there was a significant quadrant effect and a quadrant $\times$ angle effect. The pattern of bias is shown in Figure 7A and is consistent with targets in the quadrant nearest the cue $\left(270^{\circ}-360^{\circ}\right)$ being shifted toward the cue (positive bias scores) and targets in the quadrant furthest from the cue $\left(90^{\circ}-180^{\circ}\right)$ likewise being shifted toward the cue (negative bias scores).

The results of the $2 \times 2 \times 4 \times 4$ mixed factorial ANOVA conducted on the data from the three-cue condition are summarized in the Radial Bias column of Table 6. There was a significant order effect, with bias scores closer to zero when three cues were presented second $(M=-0.11, S D=$ 14.97) rather than first $(M=3.72, S D=16.38)$. Furthermore, there was a significant radius effect that was moderated by the quadrant $X$ angle interaction. The pattern of bias shown in Figure 7B does not appear to show the same type of bias toward cues as found for the one-cue condition.

\section{Discussion}

Our analyses showed pervasive cue effects on estimates of dot location in the nonrotation trials on all three measures. ${ }^{2}$ The pattern of results for angular bias was consistent with the cue-based fuzzy-boundary model, in which peripheral cues are used to establish prototypes. This cue dependence led to very different patterns of bias for the one-cue and three-cue conditions, as is shown in Figure 5. These patterns of bias were well described by our cuebased fuzzy-boundary model. There were also strong cue effects on absolute error, as is illustrated in Figure 6. These effects were consistent with the idea that cues serve to bolster the fine-grain information in regions proximal to the cue. A simple model that predicted errors as a function of the logarithmic transformation of distance to nearest cue did a good job in capturing this relationship. Finally, cues also appeared to have some effect on radial bias, but this was much less pronounced. As is shown in Figure 7, radial bias for the one-cue condition was generally consistent with a tendency to bias estimates toward the cue, but this was not the case in the three-cue condition.

A primary purpose of Experiment 2 was to examine whether the dynamic task field would lead to a cue-based frame of reference for encoding locations, rather than a cue-independent frame of reference. The critical test revolved around comparison of nonrotation trials of Experiment 2 with the trials of Experiment 1, since these were

Table 7

Parameter Values and Fit Indices for Models of Angular Bias in the Nonrotation Condition for the Three-Cues-First Data (Experiment 2)

\begin{tabular}{lcccccccc}
\hline & \multicolumn{9}{c}{ Parameter } & & $\begin{array}{c}\text { Remaining } \\
\text { Significant } \\
\text { Effects }\end{array}$ \\
\cline { 2 - 6 } Model/Radii & $p_{1}$ & $p_{2}$ & $p_{3}$ & $\lambda$ & $c$ & $R^{2}$ & \\
\hline 5 Parameters & & & & & & & \\
$\quad$ Short & $56.93^{\circ}$ & $179.48^{\circ}$ & $310.75^{\circ}$ & .717 & 0.017 & .410 & 2 \\
$\quad$ Long & $56.93^{\circ}$ & $179.48^{\circ}$ & $310.75^{\circ}$ & .717 & 0.017 & & \\
7 Parameters & & & & & & & \\
$\quad$ Short & $57.55^{\circ}$ & $179.68^{\circ}$ & $317.02^{\circ}$ & .651 & 0.015 & .597 & 1 \\
Long & $57.55^{\circ}$ & $179.68^{\circ}$ & $317.02^{\circ}$ & .930 & 0.160 & & \\
\hline
\end{tabular}

Note-The 5- and 7-parameter models are versions of the cue-based fuzzy-boundary model.

$p_{1}, p_{2}, p_{3}$, prototype values; $\lambda$, weight of fine-grain memory; $c$, sensitivity parameter. 
Table 8

Parameter Values and Fit Indices for Models of Angular Bias in the Nonrotation Condition for the Three-Cues-Second Data (Experiment 2)

\begin{tabular}{|c|c|c|c|c|c|c|c|}
\hline \multirow[b]{2}{*}{ Model/Radii } & \multicolumn{5}{|c|}{ Parameter } & \multirow[b]{2}{*}{$R^{2}$} & \multirow{2}{*}{$\begin{array}{c}\text { Remaining } \\
\text { Significant } \\
\text { Effects }\end{array}$} \\
\hline & $p_{1}$ & $p_{2}$ & $p_{3}$ & $\lambda$ & $c$ & & \\
\hline \multicolumn{8}{|l|}{5 Parameters } \\
\hline Short & $48.26^{\circ}$ & $133.00^{\circ}$ & $349.53^{\circ}$ & .991 & 0.040 & \multirow[t]{2}{*}{.367} & \multirow[t]{2}{*}{2} \\
\hline Long & $48.26^{\circ}$ & $133.00^{\circ}$ & $349.53^{\circ}$ & .991 & 0.040 & & \\
\hline \multicolumn{8}{|l|}{6 Parameters } \\
\hline Short & $120.15^{\circ}$ & - & - & .972 & 0.043 & \multirow[t]{2}{*}{.558} & \multirow[t]{2}{*}{1} \\
\hline Long & $43.00^{\circ}$ & $144.09^{\circ}$ & $349.24^{\circ}$ & .972 & 0.043 & & \\
\hline
\end{tabular}

$p_{1}, p_{2}, p_{3}$, prototype values; $\lambda$, weight of fine-grain memory; $c$, sensitivity parameter.

identical. Whereas Experiment 1 results provided clear support for the use of four quadrant-based prototypes that arise from a cue-independent frame of reference regardless of number of surrounding cues, Experiment 2 results provided clear evidence for cue-based prototypes that were dependent on the number and location of cues. Thus, our findings show that the introduction of a dynamic environment results in a dramatic shift in the location of the prototypes. This finding stands in stark contrast to the results of the study by Huttenlocher et al. (2004), who attempted to shift the location of prototypes via task-specific experience but failed to produce a change in the prototypical locations.

One implication of the present results is that it seems unlikely that the cue-independent frame of reference is automatically encoded. If it were automatically encoded, it seems likely that participants would have used it for the nonrotation trials of Experiment 2, since it would have led to reduced error. The large cue effects observed in Experiment 2 indicate that encoding targets within the cue-based frame of reference interfered with the availability of what is presumably the default encoding scheme - that is, quadrantbased encoding. These results may be consistent with the general framework of rational memory (Huttenlocher et al., 1991), assuming that failure to engage a cue-based framework would generally lead to much greater errors. These shifts are also consistent with the idea of flexibility in spatial cognition (Spencer, Simmering, \& Schutte, 2006), since participants apparently can switch from one frame of reference to another, depending on task demands.

The cue-based fuzzy-boundary model provided a very good fit to the angular bias scores in the one-cue condition (see Figure 5A). In the three-cue condition, the order in which observers experienced the cue conditions had an effect on how many prototypes were inferred by the model. When the three-cue condition occurred second, the model fit suggested the use of only one prototype for the short radius and three prototypes for the long radius (see Figure 5C). Repeated exposure to the use of just one cue may have biased participants in the second half of the experiment to use fewer cues. Because short-radius targets were farther from the cues, this tendency may have been easier to implement. When targets are closer to external cues, it may be harder to ignore those cues.

Effects of varying number of cues were not limited to angular bias but were also strongly represented in absolute error. Once again, these experimental trials are directly comparable to those of Experiment 1, except that these trials were embedded within a dynamically changing task field. The differences between Experiment 1 and Experiment 2 nonrotation trials in the effects of cues on absolute error are therefore quite striking. We used a fairly simple
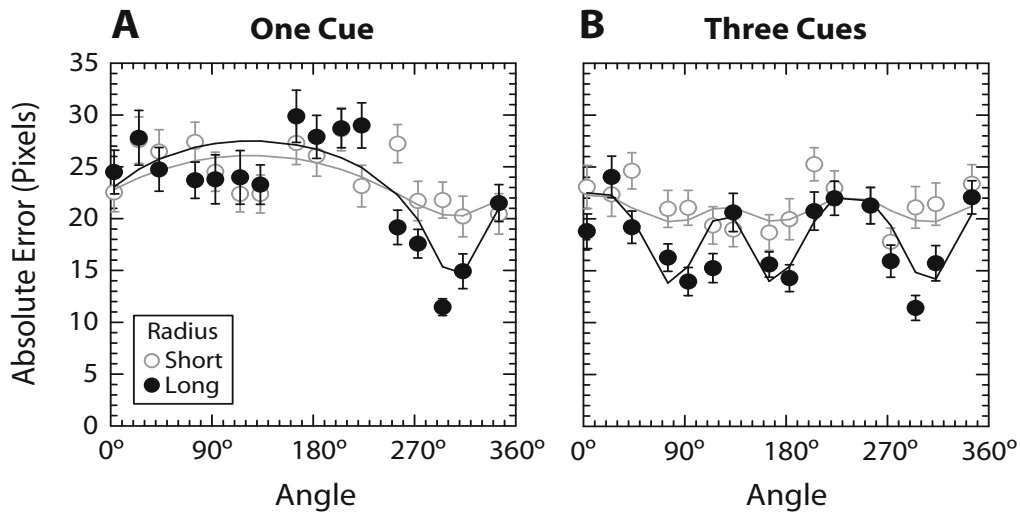

Figure 6. Fit to the mean absolute error (Experiment 2, nonrotation condition). Error bars represent one standard error, and functions show fit of model based on proximity to nearest cue. (A) The fit to the one-cue data. (B) Fit to the three-cue data. 

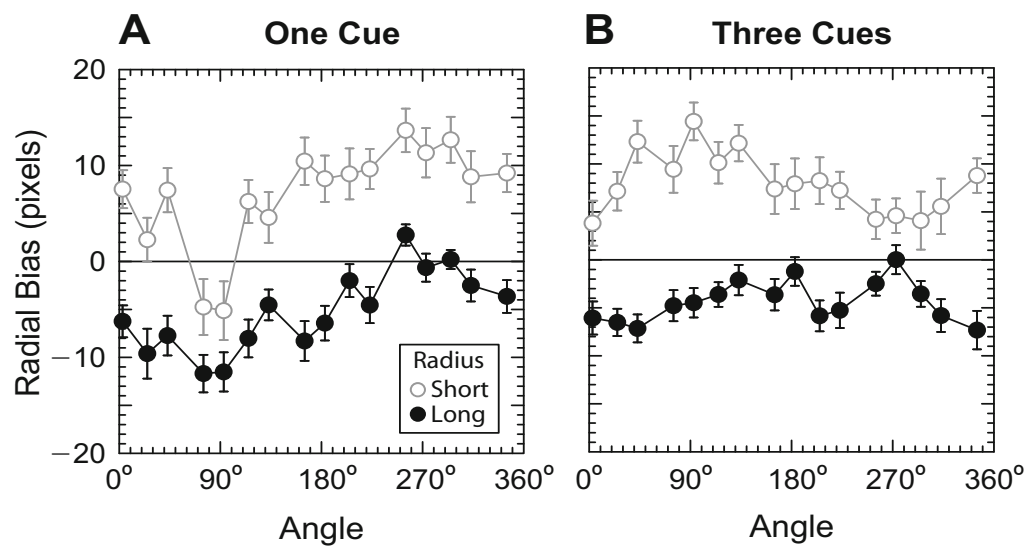

Figure 7. Mean signed radial bias (Experiment 2, nonrotation condition). Error bars represent one standard error. (A) One-cue data. (B) Three-cue data.

regression model to describe these data, regressing absolute error on the logarithm of the distance to the nearest cue. This simple approach gave a remarkably good representation of the pattern of errors for both one and three cues, as is shown in Figure 6 . The model not only captures the steep drop in error as the long-radius targets near a cue, but also captures the much reduced sensitivity to cues for the short-radius targets. Our interpretation is that many of these effects on absolute error reflect improved finegrain memory when targets are located close to cues. The cues may be conceived as anchors that stabilize memory for locations proximal to the cues, consistent with findings from studies indicating a much lower error variance near landmark cues (Werner \& Diedrichsen, 2002).

The radial bias results showed a clear tendency to place the prototype between the short- and the long-radius targets, consistent with past research (Huttenlocher et al., 1991). This pattern was quite consistent for the three-cue condition. However, in the one-cue condition, the cue itself seemed to influence the radial bias, as could be seen in the different patterns of bias across quadrants. In the quadrant nearest the cue, bias tended to be outward and thus shifted toward the cue. In the quadrant farthest from the cue, the bias was inward, meaning once again that it was shifted toward the cue.

Finally, the results of Experiment 2 provide a reasonable estimate of the power of Experiment 1 to detect cueinduced changes in the bias and error measures, because these trials were identical. The power of Experiment 1 to detect the four significant cue interaction effects on angular bias in Experiment 2 (reported in Table 3), using an $\alpha$ value of .01 , ranged from .72 to greater than .99 . Note that the mean square error terms for these effects were between three and five times larger in Experiment 2 than in Experiment 1 . Thus, even if we reduce the effect sizes observed in Experiment 2 by one fourth, the power to detect these cue interaction effects would remain quite high (averaging more than .90) in Experiment 1 because of a similar reduction in the error term. This analysis strengthens our conclusion that the lack of cue interaction effects in Experiment 1 was not due to a lack of power to detect these.

\section{GENETRAL DISCUSSION}

Our results, and those of related studies, indicate that spatial memory, even in tasks involving nothing more than specifying a location seen moments earlier, is a multifaceted phenomenon that can involve a number of different processes in response to task demands and available information. The combined results from our two experiments strongly support the position that the frame of reference used to encode spatial location is highly dependent on the stability of one's orientation to the task field. When orientation is stable, the default frame of reference appears to be cue independent, leading to spatial categories that represent the division of the visual field along vertical and horizontal axes relative to the viewer or to the display. However, when orientation shifts are introduced on the majority of trials, a cue-based frame of reference appears to be used in which available peripheral cues help define spatial prototypes used in locating the object, even on trials on which the task field was not rotated.

These results were well described by our fuzzyboundary versions of the original category-adjustment model (Huttenlocher et al., 1991). Our model builds on the basic idea of two coding mechanisms - fine-grain and categorical - but integrates the category-adjustment idea with the flexible adoption of different frame of references, such as cue-based and cue-independent frame of references. Fuzzy boundaries arise out of the idea that proximity is used to recruit those prototypes, so that objects near a boundary tend to recruit prototypes whose influence cancels out (Allen \& Haun, 2004; Haun et al., 2005; Huttenlocher et al., 1991). A useful feature of proximitybased recruitment is that it provides a way to allow prototypes to be flexibly defined as a function of cue locations. Although we believe that Experiment 2 provides strong support for the cue-based fuzzy-boundary model, other models of geometric category biases may be worth investigating in this context, such as the dynamic field theory of spatial working memory (Spencer et al., 2006).

Application of the cue-based fuzzy-boundary model to Experiment 2 provided a good account of how the pattern 
of data clearly deviated from the fixed-quadrants representation typically observed in this task (Huttenlocher et al., 1991). Our fuzzy-boundary model extends previous work by Haun et al. (2005) that modeled angular estimation and was limited to two categories. Unlike Haun et al., we did not include increased fine-grain memory weighting at the boundaries, because we did not find this necessary. Indeed, examination of error bars for stimuli near the borders show no particular increase or decrease relative to error bars for the stimuli farther from the borders (see Figures 4 and 5). This stability implies that the probabilistic recruitment of prototypes hypothesized by the fuzzyboundary Equations 3 and 4 is better represented by parallel recruitment than by serial all-or-none recruitment. If recruitment were all or none, we would expect high variability of estimates near the boundaries; sometimes estimates would be influenced in one direction by recruiting one prototype, and sometimes in the other direction by recruiting the other prototype. Instead, recruitment of prototypes appears more likely to be summed and so has a joint effect. This conclusion is supported by fitting of individual data, which mimics the findings for combined data (see Fitting, Wedell, \& Allen, 2005, for limited analysis of the data of 2 individual participants).

Results from the static task conditions of Experiment 1 provided the clearest and most convincing evidence for use of a cue-independent frame of reference in which participants simply ignore external cues. Ignoring these cues could very well be adaptive behavior, because using the cues to establish categories would have resulted in larger bias and error, as was found in the nonrotation conditions of Experiments 2. Thus, one aim of future research will be to determine the effects of adding more cues to the static environment. Even if additional cues are not used to establish prototypes, they may be used to reduce absolute error by serving as external anchors.

We also introduced a simple modeling scheme for absolute error based on logarithmic distance between the target and the nearest cue. Although absolute error is partly determined by angular bias and radial bias, it also has a component attributable to error in fine-grain memory. Our results are consistent with the conclusion that fine-grain memory for targets is bolstered in areas proximal to external cues, since these cues may serve to anchor the representation (Schmidt et al., 2003; Werner \& Diedrichsen, 2002). The success of these modeling enterprises argues for further exploration of these models in future research.

In applying findings from our study to basic principles of environmental design, we believe one implication is that the importance of external reference cues is determined by the task field. Reference cues are used in order to estimate locations in a dynamic task field, but these may be ignored in a stationary task field. Thus, for example, the number of external cues may be very important in sparse and dynamically changing environments, such as when one navigates through a parking lot or a city, so that perspectives are constantly changing. On the other hand, in settings in which orientation is relatively fixed, such as when observers look for faces in the crowd, blips on a radar screen, or objects in a visual display or in the sky, the number of external cues may have little influence on memory for spatial location. The impact of how cues in the visual environment afford the viewer a different categorical structure is an issue that needs to be considered in more detail in future research. In particular, a question that needs further investigation is the applicability of our models in a large-scale space. How do the spatial memory processes proposed by the fuzzy-boundary versions of the category-adjustment model come into play when location memory is tested in a large-scale space? This question raises interesting issues regarding the process by which observers parse the environment into categorical regions, the role of environmental geometry and peripheral cues in this parsing process, and the role of human informationprocessing limitations in cue selection and use.

Another potential application of our approach involves classic place-learning tasks, such as the Morris water maze, which focus on navigation inside a spatial array. A recent study indicated that the extension of the fuzzyboundary model is applicable to memory for incline and azimuth from the viewer's perspective (Haun et al., 2005). In the present study, we employed a task that was comparable in some ways to the Morris water maze (Morris, 1981), but clearly there were differences. Navigation is involved in the Morris water maze, but it is not involved in our task. The Morris water maze typically involves a single place to be learned; our task involved hundreds of trials with different locations to be remembered. Furthermore, the Morris water maze, as a task performed in a spatial array with an actual view as a navigator inside the maze, contrasts with an allocentric point of view. Thus, the results from our experiment are more suggestive than conclusive when addressing the question of whether categorical bias is present in animal and human behavior in this classic place-learning paradigm. In future research, we hope to obtain more conclusive evidence with humans using these different approaches. However, it should be noted that a recent human analogue study of the Morris water maze supports the idea that cues may be used as prototypes in a vista space, with memory for locations being biased toward the nearest available environmental cue and the magnitude of bias being a function of distance to the available cue (Fitting, Allen, \& Wedell, 2008).

\section{AUTHOR NOTE}

The authors thank Lena Fitting Kourkoutis for her help with data transformation procedures. Correspondence concerning this article should be addressed to S. Fitting, Department of Psychology, University of South Carolina, Columbia, SC 29208 (e-mail: fitting@sc.edu).

\section{REFERENCES}

Allen, G. L., \& Haun, D. B. M. (2004). Proximity and precision in spatial memory. In G. L. Allen (Ed.), Human spatial memory: Remembering where (pp. 41-63). Mahwah, NJ: Erlbaum.

Allen, G. L., Kirasic, K. C., Rashotte, M. A., \& Haun, D. B. M. (2004). Aging and path integration skill: Kinesthetic and vestibular contributions to wayfinding. Perception \& Psychophysics, 66, 170-179.

Batschelet, E. (1981). Circular statistics in biology. New York: Academic Press.

Burgess, N., Spiers, H. J., \& Paleologou, E. (2004). Orientational manoeuvres in the dark: Dissociating allocentric and egocentric influences on spatial memory. Cognition, 94, 149-166. 
Cheng, K. (1986). A purely geometric module in the rat's spatial representation. Cognition, 23, 149-178.

CoOK, R. G., \& TAURo, T. L. (1999). Object-goal positioning influences spatial representation in rats. Animal Cognition, 2, 55-62.

EASTON, R. D., \& SHOLL, M. J. (1995). Object-array structure, frames of reference, and retrieval of spatial knowledge. Journal of Experimental Psychology: Learning, Memory, \& Cognition, 21, 483-500.

Fitting, S., Allen, G. L., \& Wedell, D. H. (2008). Remembering places in space: A human analog study of the Morris water maze. In T. Barkowsky, M. Knauff, G. Ligozat, \& D. R. Montello (Eds.), Spatial cognition V (Lecture Notes in Computer Science, Vol. 4387, pp. 59-75). Berlin: Springer.

Fitting, S., Wedell, D. H., \& Allen, G. L. (2005). Memory for spatial location: Influences of environmental cues and task field rotation. In A. G. Cohn \& D. M. Mark (Eds.), COSIT 2005 (Lecture Notes in Computer Science, Vol. 3693, pp. 459-474). Berlin: Springer.

Gallistel, C. R. (1990). The organization of learning. Cambridge, MA: MIT Press.

Greenhouse, S. W., \& Geisser, S. (1959). On methods in the analysis of profile data. Psychometrika, 24, 95-112.

Hartley, T., Trinkler, I., \& Burgess, N. (2004). Geometric determinants of human spatial memory. Cognition, 94, 39-75.

Haun, D. B. M., Allen, G. L., \& Wedell, D. H. (2005). Bias in spatial memory: A categorical endorsement. Acta Psychologica, 118, 149-170.

Hermer, L., \& Spelke, E. S. (1996). Modularity and development: The case of spatial reorientation. Cognition, 61, 195-232.

Huttenlocher, J., Hedges, L. V., Corrigan, B., \& Crawford, L. E. (2004). Spatial categories and the estimation of location. Cognition, 93, 75-97.

Huttenlocher, J., Hedges, L. V., \& Duncan, S. (1991). Categories and particulars: Prototype effects in estimating spatial location. Psychological Review, 98, 352-376.

Kamil, A. C., \& Cheng, K. (2001). Way-finding and landmarks: The multiple-bearings hypothesis. Journal of Experimental Biology, 204, 103-113.

Montello, D. R., Richardson, A. E., Hegarty, M., \& Provenza, M. (1999). A comparison of methods for estimating directions in egocentric space. Perception, 28, 981-1000.

Morris, R. G. M. (1981). Spatial localization does not require the presence of local cues. Learning \& Motivation, 12, 239-260.

Morris, R. G. M., Garrud, P., Rawlins, J. N. P., \& O'Keefe, J. (1982). Place navigation impaired in rats with hippocampal lesions. Nature, 297, 681-683.

Morris, R. G. [M.], \& PArslow, D. M. (2004). Neurocognitive components of spatial memory. In G. L. Allen (Ed.), Human spatial memory: Remembering where (pp. 217-247). Mahwah, NJ: Erlbaum.

Mou, W., \& McNamara, T. P. (2002). Intrinsic frames of reference in spatial memory. Journal of Experimental Psychology: Learning, Memory, \& Cognition, 28, 162-170.

Mou, W., McNamara, T. P., Valiquette, C. M., \& Rump, B. (2004). Allocentric and egocentric updating of spatial memories. Journal of Experimental Psychology: Learning, Memory, \& Cognition, 30, 142157.

NADEL, L. (1990). Varieties of spatial cognition: Psychobiological considerations. In A. Diamond (Ed.), The development and neural bases of higher cognitive functions (Annals of the New York Academy of Sciences, Vol. 608, pp. 613-636). New York: New York Academy of Sciences.

Nelson, T. O., \& Chaiklin, S. (1980). Immediate memory for spatial location. Journal of Experimental Psychology: Human Learning \& Memory, 6, 529-545.
O'Keefe, J., \& Nadel, L. (1978). The hippocampus as a cognitive map. Oxford: Oxford University Press, Clarendon Press.

Schmidt, T., Werner, S., \& DiedrichSEN, J. (2003). Spatial distortions induced by multiple visual landmarks: How local distortions combine to produce complex distortion patterns. Perception \& Psychophysics, 65, 861-873.

Schutz, R. W., \& Roy, E. A. (1973). Absolute error: The devil in disguise. Journal of Motor Behavior, 5, 141-153.

SHEPARD, R. N. (1987). Toward a universal law of generalization for psychological science. Science, 237, 1317-1323.

Shepard, R. N., \& Metzler, J. (1971). Mental rotation of threedimensional objects. Science, 171, 701-703.

Spencer, J. P., Simmering, V. R., \& Schutte, A. R. (2006). Toward a formal theory of flexible spatial behavior: Geometric category biases generalize across pointing and verbal response types. Journal of Experimental Psychology: Human Perception \& Performance, 32, 473-490.

Tolman, E. C. (1948). Cognitive maps in rats and men. Psychological Review, 55, 189-208.

WANG, R. F., Hermer, L., \& Spelke, E. S. (1999). Mechanisms of reorientation and object localization by children: A comparison with rats. Behavioral Neuroscience, 113, 475-485.

WANG, R. F., \& SPELKE, E. S. (2000). Updating egocentric representations in human navigation. Cognition, 77, 215-250.

Wedell, D. H., Fitting, S., \& Allen, G. L. (2007). Shape effects on memory for location. Psychonomic Bulletin \& Review, 14, 681-686.

WERNER, S., \& DIEDRICHSEN, J. (2002). The time course of spatial memory distortions. Memory \& Cognition, 30, 718-730.

WILKINSON, L. (1989). SYSTAT: The system for statistics. Evanston, IL: SYSTAT.

\section{NOTES}

1. One way to avoid the positing of virtual categories would be to reframe the model within circular statistics in which the $x-y$ vector of coordinates is replaced with a cosine-sine vector of coordinates. Circular statistics based on this alternative vector representation are particularly useful in computing means and standard deviations of raw angles (Batschelet, 1981). Because our statistical analyses will be computed on angular bias, rather than on raw angles directly, linear statistics can be appropriately applied. Furthermore, a problem with using the vector representation and circular statistics within Equations 3 and 4 is that proper computation of the signed angular distance is conditional on the respective quadrants of the vectors. We have found that nonlinear estimation using conditional statements is problematic. Instead, the use of the exponential similarity function, in which the influence of a prototype is exponentially diminished with distance, results in any location's being influenced essentially by the pull of prototypes from the two adjacent categories. Finally, as was developed in the section on the cue-based fuzzy-boundary model, the case of a single category prototype can be handled easily using the idea of virtual prototypes and leads to the appropriate predicted biases modeled later in Experiment 2.

2. Although we are not reporting the analyses from the rotation trials in this article, it should be noted that the usable data from these trials demonstrated extremely large cue-based effects on angular bias and absolute error. These effects tended to grow as rotation increased. We focus on the nonrotation trials, since these provide the clearest comparison with the identical trials used in Experiment 1.

(Manuscript received May 18, 2006; revision accepted for publication January 9, 2007.) 